\title{
Interacting with biological membranes using organic electronic devices
}

Josefin Nissa 



\title{
Interacting with biological membranes using organic electronic devices
}

\author{
Josefin Nissa
}

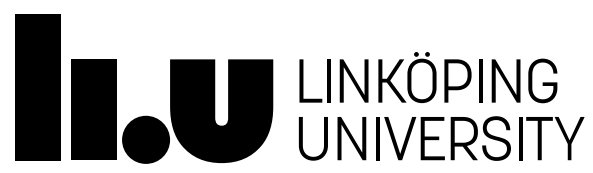

Laboratory of Organic Electronics, Department of Science and Technology

Norrköping 2020 
(c) EY-NC This work is licensed under a Creative Commons AttributionNonCommercial 4.0 International License.

https://creativecommons.org/licenses/by-nc/4.0/

\section{Interacting with biological membranes using organic electronic devices}

Josefin Nissa

During the course of the research underlying this thesis, Josefin Nissa was enrolled in Forum Scientium, a multidisciplinary graduate school at Linköping University, Sweden.

Linköping Studies in Science and Technology. Dissertation No. 2118

Copyright (C) 2021 Josefin Nissa, unless otherwise noted.

Printed by LiU-Tryck, Linköping 2021

ISBN: 978-91-7929-712-1

ISSN: 0345-7524

Electronic publication: http://www.ep.liu.se 


\section{Cover art}

2D FFT filtered data from AFM image recorded in air. Sample: silica mesoporous thin film exposed to POPC vesicles and gently rinsed in DI water. Original image shown below. More information about the substrate can be found in Paper II.

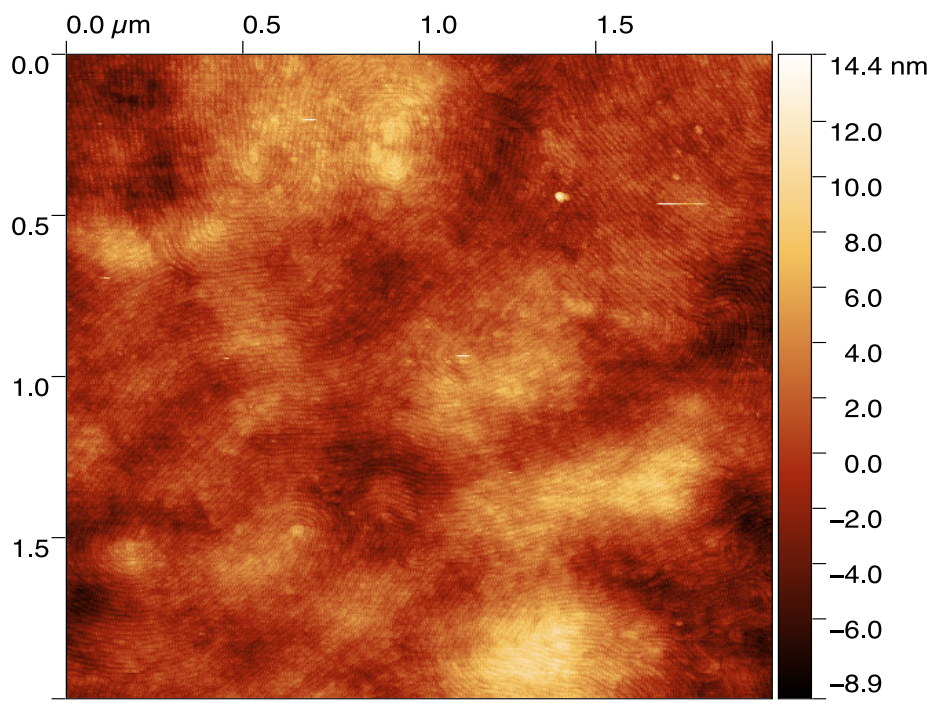





\section{Abstract}

Many physiological processes are reliant on activities in the cell membrane. These activities are of great importance to our well-being since they allow the cells to respond to their environment and communicate with each other to coordinate their function as tissues and organs. In this thesis the use of organic electronic devices to interface with cell membranes has been explored. Organic electronics are especially suited for the task given their ability to transduce ionic to electronic signals. Four scientific papers are included in the thesis, where organic electronic devices were used together with living cells and supported lipid bilayers (SLB).

In the first paper a ferroelectric cell release surface was presented. Release of cells cultured on the surface was induced by a polarization change in the ferroelectric polymer. This non-enzymatic release method was developed primarily for treatment of severe burns.

The remaining three papers strived to combine lipid bilayers and the conjugated polymer poly(3,4-ethylenedioxythiophene) doped with polystyrene sulfonate (PEDOT:PSS) in biosensors. The target device was an organic electrochemical transistor (OECT) functionalized with a supported lipid bilayer. Several aspects of the integration were explored, including promotion of vesicle fusion onto PEDOT:PSS and optimization of OECT design and biasing conditions for sensing.

For SLB formation on PEDOT:PSS two different silica material systems, one PEDOT:PSS/silica composite and one mesoporous silica film, were evaluated with respect to electrical properties and quality of the resulting bilayer. The electrical properties were found to be similar, but the quality of the bilayer was better on the mesoporous silica film.

In the last two papers the focus was on optimization of OECTs for sensing purposes. Biasing conditions for operation at high transconductance were identified, as well as design principles for large sensor output in impedance sensing. 


\section{Populärvetenskaplig sammanfattning}

I cellmembranet sker många viktiga processer som styr vår kroppsfunktion. Proteiner knutna till membranet ser till att celler kan kommunicera med varandra, ta upp näring, göra sig av med avfall och utföra sina specifika uppgifter. Ett protein som inte fungerar som det ska kan göra oss sjuka och många läkemedel riktar in sig på just membranproteiner för att göra dem mer eller mindre aktiva. I den här avhandlingen studeras hur organisk elektronik kan kombineras med olika typer av cellmembran för att på sikt utveckla biosensorer och verktyg för cell- och vävnadsodling. Sensorerna skulle exempelvis kunna användas för att snabbt testa om ett läkemedel har avsedd effekt på proteinet.

Organisk elektronik är elektronik baserad på polymerer - det som vi i dagligt tal kallar plast. I avhandlingen används två olika polymerer, en som leder ström och en som är ferroelektrisk. Den ferroelektriska polymeren kan ges en positiv eller negativt laddad yta med hjälp av ett elektriskt fält. Genom att ändra riktningen på det elektriska fältet går de att byta mellan positiv och negativ yta. Laddningen stannar kvar även när det elektriska fältet försvinner. I en av avhandlingens artiklar användes den ferroelektriska polymeren till en yta för att odla celler. När ytladdningen sedan ändrades släppte cellerna från ytan och kunde flyttas till en ny växtplats. Processen involverar inga skadliga kemikalier eller skrapning som kan skada cellerna. Redskap för att flytta celler och odlad vävnad utan att de tar skada spås bli en viktig komponent för att i framtiden kunna odla organ istället för att ta dem från donatorer. I just det här fallet var målet en behandling av svåra brännskador.

De övriga artiklarna fokuserar på att kombinera cellmembran och en ledande polymer för att göra biosensorer. I avhandlingens andra artikel undersöktes hur vesiklar - små sfärer av cellmembran - kan fås att forma ett kontinuerligt membran ovanpå den ledande polymeren. För att underlätta processen användes tillskott av kiseloxid, både i form av små (ca $30 \mathrm{~nm}$ i diameter) kulor som blandades med polymeren och som ett poröst lager som lades ovanpå polymeren. Att oxidlagret släpper igenom joner är viktigt eftersom jonerna kan styra ledningsförmågan hos polymeren. Om inga joner kommer igenom kan materialsystemet inte användas till sensorer.

De två återstående artiklarna handlar om hur elektrokemiska transistorer baserade på den ledande polymeren kan optimeras för att ge hög sensorsignal. I transistorer går en ström i kanalen mellan två av transistorns kontakter. Den tredje kontakten, grinden, reglerar ledningsförmågan i kanalen, och därmed hur stor strömmen är. Optimeringen som gjorts handlar dels om att få det inbördes storleksförhållandet 
mellan transistorns grind och dess kanal rätt, och också hur transistorn bör spänningssättas för att få så stor utsignal som möjligt från sensorn. Slutsatserna kan komma till användning i flera olika typer av biosensorer och gäller inte bara för mätningar som involverar cellmembran. 


\section{Acknowledgements}

Even though the path here has felt lonely from time to time and there is only one name on the cover of this book, there has been many people around me helping me and carrying me forward. In fact, you are so many, so I won't even try to mention you all here. Some people have been standing out a little extra and I would like the world to know.

First of all, I would like to thank my supervisors. Magnus, for giving me the opportunity, and always seeing the big picture and instinctively knowing where to look for the missing pieces to fill gaps in the knowledge. Daniel, for your neverending optimism and for letting me find my own ways.

I would also like to thank all my co-authors for actually doing the science together with me. Especially Susanna, Henrik, Hanna, Hudson and Pelle. Fredrik, for your role in the dreamer team starting up the project. And, of course, Ek, thank you for truly getting me started in the lab.

Since I have been doing experimental research it has been essential to have access to functioning labs, so thank you Anna, Lasse, Putte, Thomas and Meysam for keeping the labs open, and the equipment up and running. I would also like to thank our administrators over the years, for making this journey smoother and always seeming to know everything there is to know about travels and rules. Jens, thank you for running the lab IT and setting up the best remote solutions.

During my PhD I've had the pleasure of being part of the Laboratory of Organic Electronics along with a fantastic mix of people. Thank you all for brightening the days. I would also like to thank all members of Forum Scientium for creating such a friendly and helping atmosphere and broadening perspectives. Theresia, let's start our lunch habit back up as soon as we can. We all miss you.

I also have a lot of important people outside the university. One of the best days of the week has always been Wednesdays when I get to meet with my choir, Bel Canto. I hope we can all sing together again soon. And my friends from Lund: Caroline, Christian, Matilda, Robert and Simon, thank you for the new year's celebrations and the laughs. And the occasional whining too.

Finally, I need to express my gratitude towards my family. Mamma och pappa, I know standing on the side has sometimes been hard on you, too. But you have equipped me with a high base-level confidence and even though it has taken some hits and wear, it has kept me on my feet. I hope you can enjoy and celebrate the end 
result with me. My sister Karolin, and Emil, for making the move here seem like a smaller step.

David, thank you for always seeing me and telling me the things I already know but still need to hear to believe. Thank you for bringing the adventure, outdoors and within. I also need to thank our unborn child, for setting a very hard deadline for the work with this thesis. I can't wait to meet you and earn an even greater title. 


\section{List of papers included in the thesis}

\section{Paper I}

Ferroelectric surfaces for cell release

Henrik Toss, Susanna Lönnqvist, David Nilsson, Anurak Sawatdee, Josefin Nissa, Simone Fabiano, Magnus Berggren, Gunnar Kratz, Daniel T. Simon

Synthetic Metals 2017, 228, 99-104

Contribution: Optimization of material processing and measurement setup. Took part in the editing of the manuscript. Did not contribute to the care for cells and cell image analysis.

\section{Paper II}

Formation of supported lipid bilayers derived from vesicles of various compositional complexity on conducting polymer/silica substrates

Hanna Ulmefors, Josefin Nissa, Hudson Pace, Olov Wahlsten, Anders Gunnarsson, Daniel T. Simon, Magnus Berggren, Fredrik Höök

\section{Submitted}

Contribution: Experimental work for determination of electrical properties and roughness of produced films. Material and process optimization to ensure stability during operation. Wrote parts of the first draft and contributed to the final editing of the manuscript. 


\section{Paper III}

The role of relative capacitances in impedance sensing with organic electrochemical transistors

Josefin Nissa, Per Janson, Magnus Berggren, Daniel T. Simon

\section{Submitted}

Contribution: All computer modelling and experimental work, except transistor design and fabrication. Wrote most of the first draft and contributed to the final editing of the manuscript.

\section{Paper IV}

Expanding the understanding of organic electrochemical transistor function

Josefin Nissa, Per Janson, Daniel T. Simon, Magnus Berggren

\section{Submitted}

Contribution: Initial trend observation and idea. All computer modelling and experimental work, except transistor design and fabrication. Wrote most of the first draft and contributed to the final editing of the manuscript. 


\section{Contents}

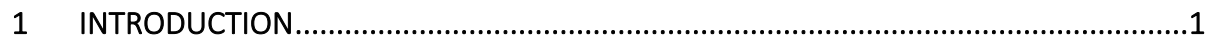

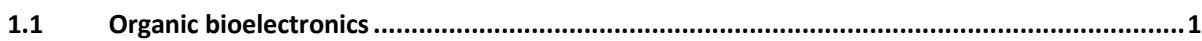

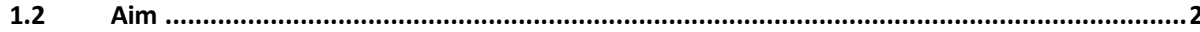

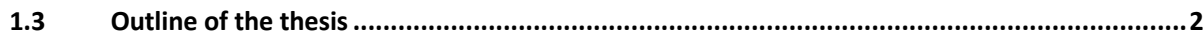

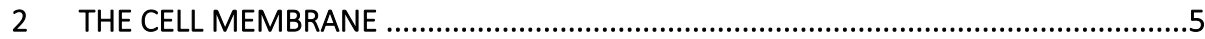

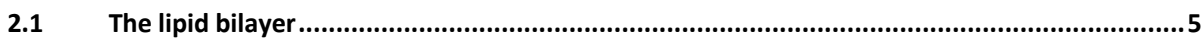

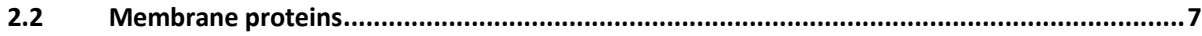

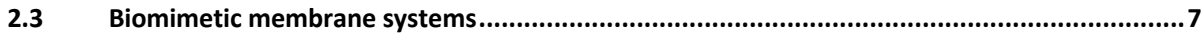

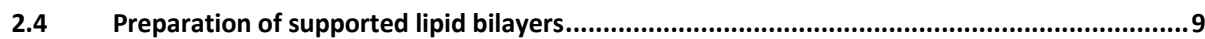

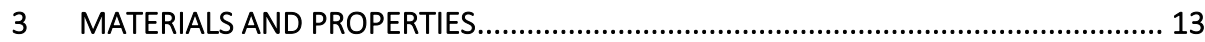

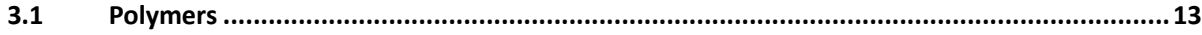

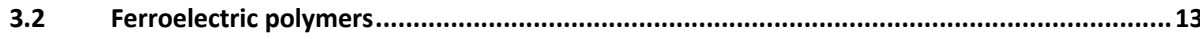

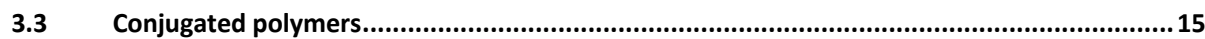

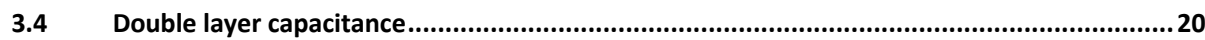

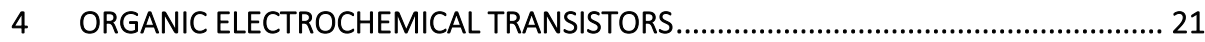

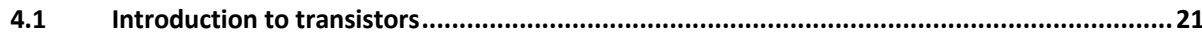

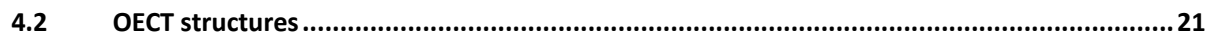

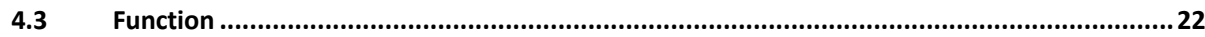

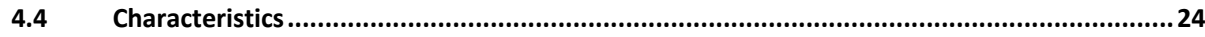

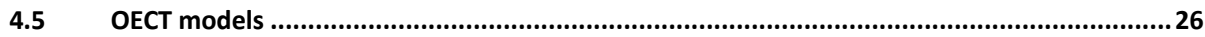

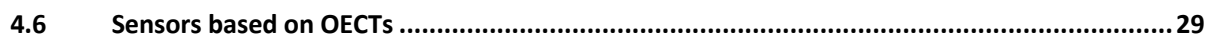




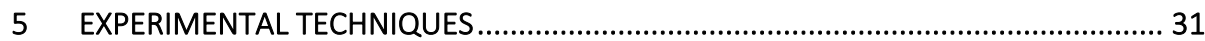

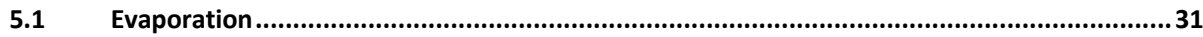

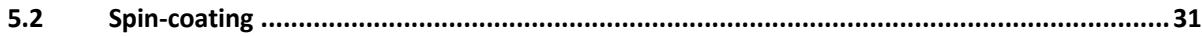

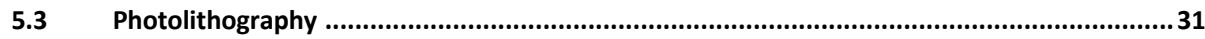

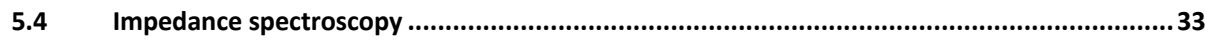

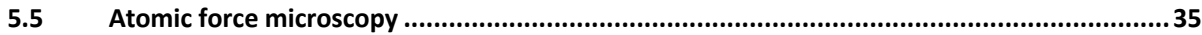

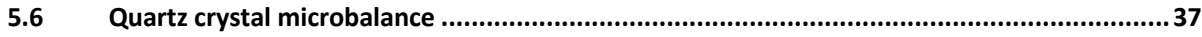

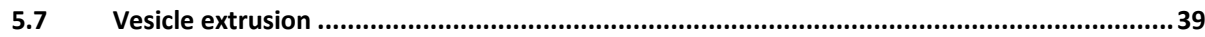

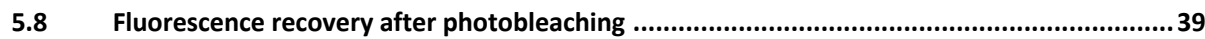

5.9 Electrical characterization of transistors ........................................................................... 40

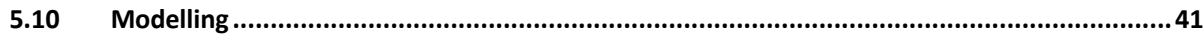

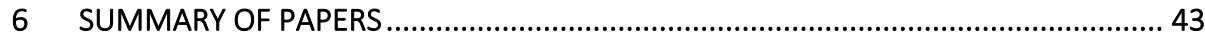

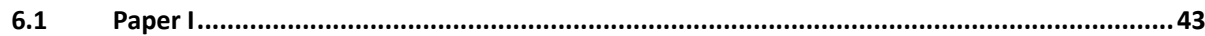

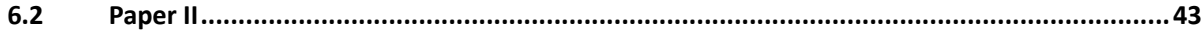

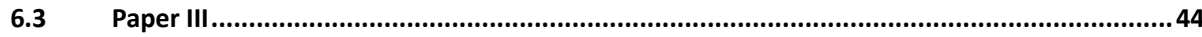

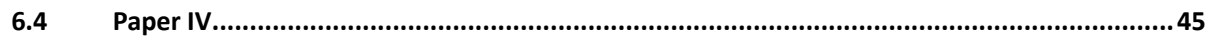

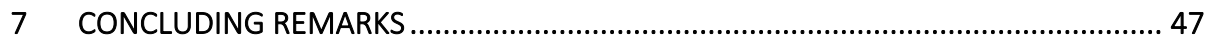

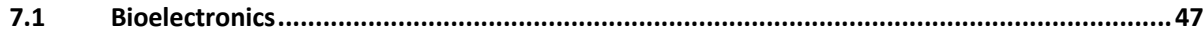

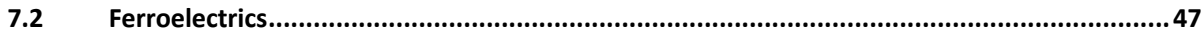

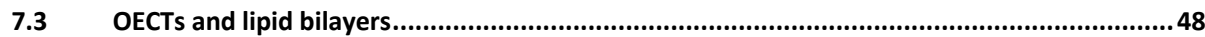

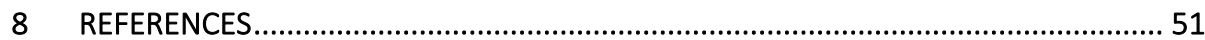

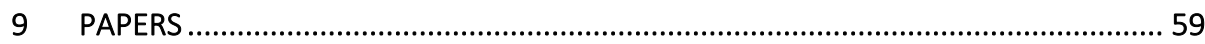





\section{Introduction}

\subsection{Organic bioelectronics}

In our daily lives we are surrounded with electronic equipment, in our mobile phones and cars as well as in traffic lights, and automatic door openers. Many of these gadgets have sensors to collect information from the surroundings. This information is processed and analyzed before being presented to the user, or an action is triggered. With technology helping us in so many parts of life already, the step to involving technology further in our medical diagnostics and health care does not seem too long. Of course, medicine is not being carried out in a technology vacuum today, patients are already getting help from pacemakers, ventilators and blood glucose meters, and doctors have access to a wide range of imaging techniques. As for lab diagnostics, the now dying year of 2020 was the year when PCR (for polymerase chain reaction) became a term known to the general public as a step in the confirmation of ongoing COVID-19 infection. Still, many of us probably wondered if there was an easier, faster and cheaper way to test for the infection. And there are other goals with the potential to revolutionize people's lives that scientists around the world are working towards. Some examples could be labgrown organs to eliminate the need for matching donors to treat severe diseases (1), devices to bypass or stimulate use of alternative pathways around spinal cord injuries $(2,3)$, or more constant monitoring of vitals to catch indicators of a disease before it manifests symptoms (4).

To achieve these goals there is a need for an interface between the biological system and the electronic equipment that is most likely going to be responsible for the monitoring, control and communication of data. There is a need for sensors that can detect relevant changes, and actuators and drug delivery systems that can respond. Depending on the application we need materials that are bio-compatible and stable over long periods of time. The sensors and actuators need to perform in water and the interfaces need to offer a good translation between biological and electrical signals. This translation is where the organic bioelectronics can offer a good solution. The body uses both electrical and chemical signaling, and the organic electronic materials are capable of this dual mode of operation as well (5). Organic electronic devices have been used to differentiate stem cells in cell cultures (6), deliver precise amounts of medicine (7) and are promising platforms for biosensors (8). They can also be incorporated in flexible devices, opening for use within wearable technology and long-term implants (9). Another advantage is that many of 
the materials can be printed, meaning production volumes for devices can be large bringing costs down (10).

In this thesis the efforts to establish communication between organic electronic devices and biological systems are focused to the cell membrane. The main reason is that the cell membrane is where the cell itself receives information from the rest of the body and displays proteins for anchoring and recognition, allowing tissues and organs to function properly. Additionally, the characteristics of the cell membrane acting as a semipermeable ion barrier offers interesting opportunities for using it as a sensor element in combination with conducting polymers. The interest in the cell membrane as an ion blocker stems from the interaction between conducting polymers and ions in the electrochemical doping and de-doping of the polymer. The organic electrochemical transistor (OECT) is a device relying on ion exchange and is able to translate ionic signals to electronic (11). Proteins in the cell membrane can change the overall ion permeability in response to external stimuli. A sensor system able to detect these changes could find applications within drug development or diagnostics. Such a platform could also be used for research on protein function and protein-related diseases.

A cell culture and controlled release surface incorporating a ferroelectric polymer is proposed and evaluated in Paper I. This work was done with applications within tissue engineering in mind. In Paper II two material systems to promote vesicle fusion on a conducting polymer were investigated. The last two papers included in the thesis (III and IV) cover optimization of OECT design and operation parameters for biosensing applications.

\subsection{Aim}

The aim of the work presented within the thesis has been to develop and characterize organic material systems and devices that can be used for interactions with cell membranes. Living cells as well as cell membrane model systems have been included. The research has to some extent been technical in its nature, meaning it has been performed with a certain goal or application in mind, and the efforts have been focused on finding ways to improve performance or overcome key obstacles along the way. The aim and outcome of each of the included papers is presented both in the paper itself, and in the summary of papers provided in Chapter 6 .

\subsection{Outline of the thesis}

The thesis is divided into two parts. The scientific work included in this thesis is presented in the scientific papers in the second part of the thesis. The first part is intended as introduction to the materials and concepts applied in the scientific 
papers. This introductory part is meant to help readers understand the papers. The explanations of material properties, concepts and techniques have been tailored to cover the aspects important for the scientific papers and are not exhaustive. In the first part, the chapters cover the cell membrane, organic materials and their properties and experimental techniques. Special attention is given to the organic electrochemical transistor. There is also a short summary of the major results and conclusions from the papers. Finally, some concluding remarks are made, putting the work in a bigger perspective and suggesting directions for future work. 



\section{The cell membrane}

All living cells are surrounded by a cell membrane, keeping the cell's interior separated from the exterior environment. The main constituents of the membrane are lipids and proteins. The lipids form a lipid bilayer, gathering the hydrophobic tails in the centre of the bilayer and the hydrophilic heads on its surfaces. The hydrophobic core prevents free diffusion of ions and large watersoluble compounds across the membrane making the membrane an effective barrier layer (12).

Proteins are embedded in the cell membrane and are responsible for several vital cell functions. With the help of proteins, nutrients and waste products can be transported in and out of the cell. Proteins are also responsible for communication between cells by detection of chemical signals. Figure 2.1 shows a schematic of a cell with its cell membrane and some different cell membrane functions.

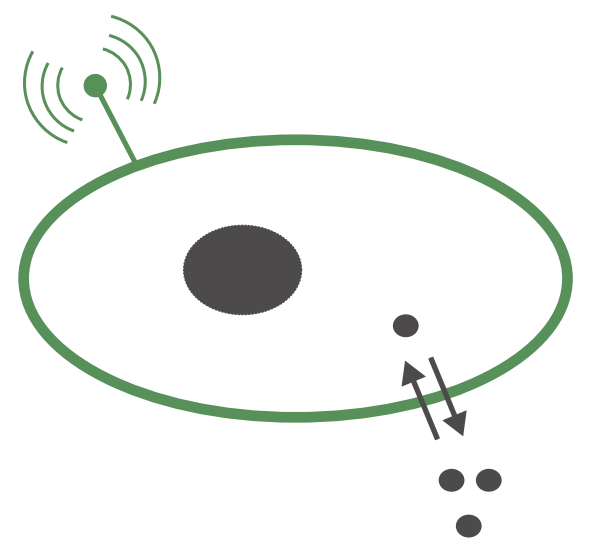

Figure 2.1 The cell membrane separates the cell's interior from the exterior. Proteins embedded in the membrane are vital for transport in and out of the cell and communication between cells.

\subsection{The lipid bilayer}

The lipids in the cell membrane have a hydrophilic head and one or two hydrophobic fatty-acid tails. Since both the interior and exterior environments of the cell are aqueous, the most energetically favorable configuration for the lipids is a double layer where the hydrophobic tails are shielded from the surrounding water. Figure 2.2 shows an illustration of section of a lipid bilayer. 


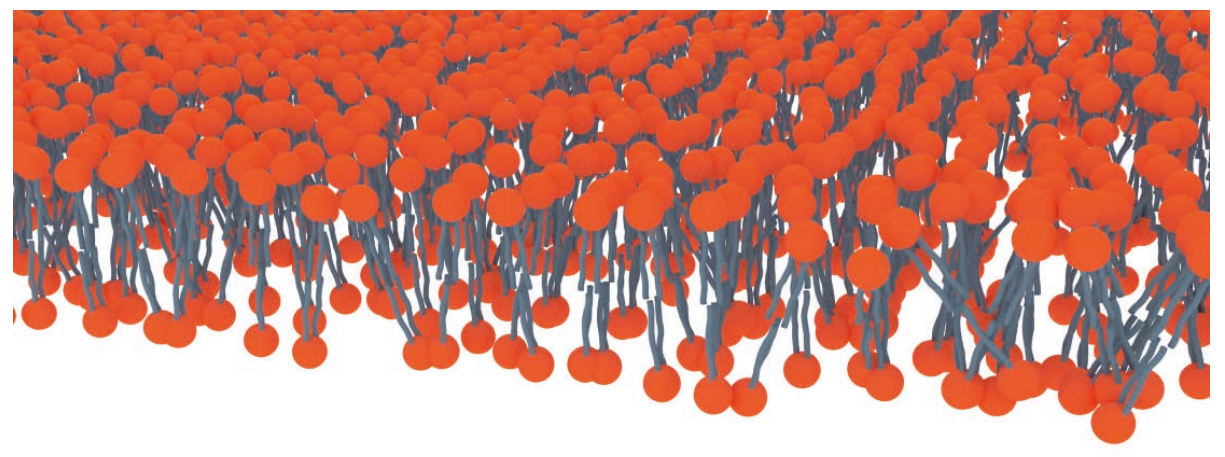

Figure 2.2 3D illustration of a lipid bilayer.

The most abundant lipid type in the cell membrane are the glycerophospholipids, where a glycerol and phosphate group link the hydrophilic head to the hydrophobic tails (13). Most common among the phospholipids are the phosphatidylcholines, with choline as hydrophilic head and two fatty-acid tails. Figure 2.3 shows the structure of the phospholipid 1-palmitoyl-2-oleoyl-snglycero-3-phosphocholine (POPC) used in Paper II

The lipids in the bilayer move around through diffusion, with the diffusion rate being determined mainly by the interactions between the hydrophobic tails. Different cells have different lipid compositions, giving each cell membrane unique properties. The lipid composition also varies within a cell membrane, creating functional regions of the membrane (14).

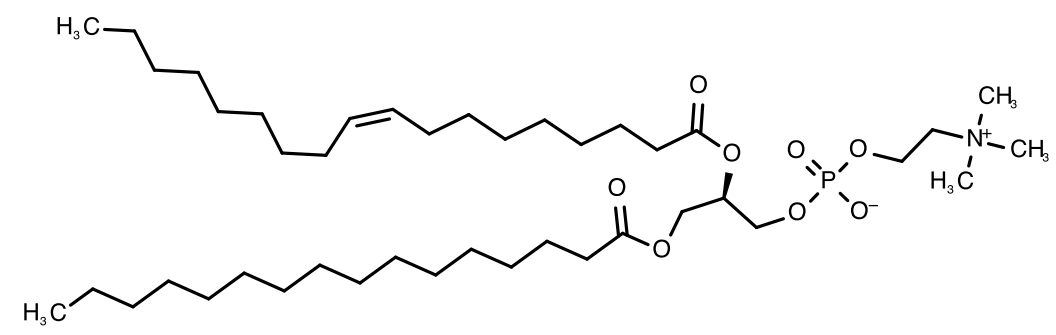

Figure 2.3 POPC chemical structure 


\subsection{Membrane proteins}

Proteins are molecules constructed from amino-acids, with the amino-acid sequence being encoded in our DNA (15). About 20-30\% of an organism's genome is estimated to code for membrane proteins (16). The proteins associated with the cell membrane can be bound to the membrane in various ways. Some are transmembrane proteins, meaning they extend across the membrane. Like the lipids, these proteins typically have both hydrophilic and hydrophobic segments which control how the protein associates with the membrane (17). Others reside in just one of the bilayer leaflets, displaying an active part either on the inside or outside of the cell. Some membrane proteins are anchored to lipids, extending out from the membrane, and yet others are attached to other membrane proteins (12). The functions of the proteins vary, but the intended targets for the work presented here has been mainly channel proteins. Some channels are always open, while others open and close as a response to external stimuli, such as changes in the potential across the cell membrane, heat, or the binding of a ligand to the channel protein. Channel proteins are, for example, important for the functions of neurons since they help convey signals across the synaptic cleft and propagate the action potential along the axon of the neuron (18). The channels are also essential to muscle contraction (19). Transporters actively shuttle compounds across the membrane. One example of a transporter is the glucose transporter that actively brings glucose into the cell while consuming chemical energy stored in adenosine triphosphate (ATP). The transporters can also utilize concentration gradients to propel the transport. Yet other membrane proteins are enzymes that catalyze chemical reactions.

\subsection{Biomimetic membrane systems}

This section will give an overview of different types of cell membrane models available to scientists who study various properties of the cell membrane components.

\subsubsection{Black lipid membranes}

The black lipid membranes were the first demonstrations of artificial cell membranes. Black lipid membranes are formed by painting lipids in an organic solvent over a hole in a barrier separating two compartments filled with aqueous solutions (20). The lipid membrane then covers the orifice as illustrated in Figure 2.4. Already in the earliest publications the same voltage-dependent behaviour that had been observed in frog nerves, called action potentials, were seen in these reconstituted membranes when equipped with proteins isolated from frog neurons (21). The black lipid membranes exhibit the highest 
resistivities among the biomimetic lipid membranes, reaching values of $100 \mathrm{M} \Omega \mathrm{cm}^{2}$ (22).

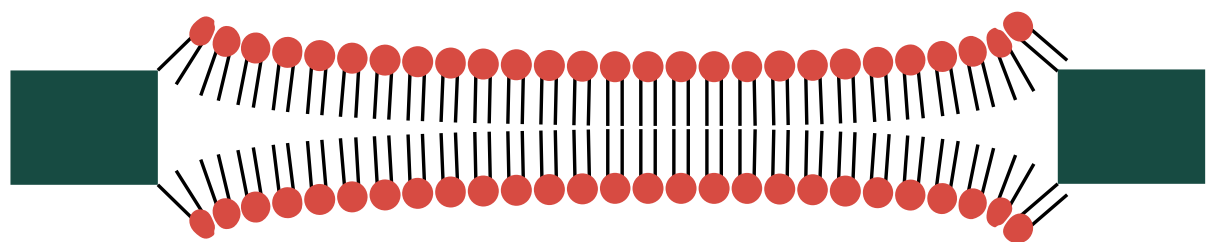

Figure 2.4 Cross-sectional view of a black lipid membrane spanning an orifice.

\subsubsection{Supported lipid bilayers}

Lipid bilayers can be formed on top of solid substrates, making them compatible with standard device fabrication and common microscopy techniques (23). There are several names for this type of membrane, the supported lipid bilayer (SLB) used throughout this thesis being one of them. Other options are supported bilayer lipid membrane (sBLM) and supported phospholipid bilayer (SPB). The bilayer is formed on the surface of the substrate, with a thin hydration layer between substrate and the hydrophilic heads of the lipid.

The supported lipid bilayers can be anchored to the substrate surface by adding linkers to the hydrophilic heads. These membranes are called tethered membranes. A tether can add a little extra space between the substrate surface and the bilayer or promote the formation of the bilayer on substrates that are otherwise difficult to work with (24). Space added between the bilayer and substrate by a linker can be important for retaining function of membrane proteins protruding out from the SLB.

\subsubsection{Vesicles}

A vesicle is a spherical liposome that has a structure similar to that of the cell membrane with the hydrophilic tails of the lipids sticking together in a middle layer and the hydrophilic headgroup facing the outside and center of the vesicle as illustrated in Figure 2.5. Vesicles occur naturally in cells and are vital for several physiological functions, such as release of neurotransmitters into the synaptic cleft in the nervous system (25) and addition of new lipids into the cell membrane (26). There are also extracellular vesicles taking part in communication and coordination between cells (27). Vesicles are studied as vessels for drug delivery, with the molecule of interest being loaded in the aqueous center compartment (28). 

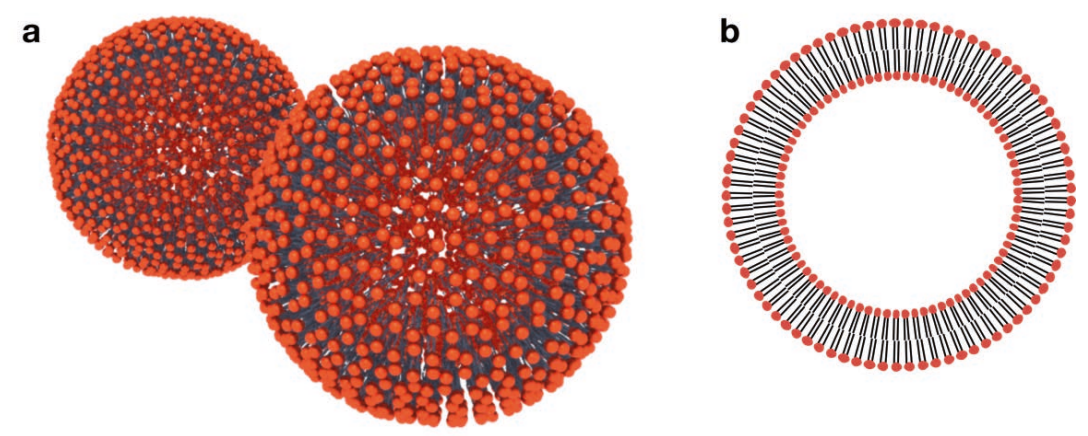

Figure 2.5 Illustrations of lipid vesicles. a) 3D illustration b) cross-sectional view.

\subsection{Preparation of supported lipid bilayers}

\subsubsection{Vesicle fusion}

Allowing vesicles to adsorb and rupture on a solid support results in a supported lipid bilayer (29). The process is illustrated in Figure 2.6. Vesicle fusion has a high success rate on glass and mica. In the presence of calcium ions there is also spontaneous rupture of anionic vesicles on ITO (30). Among the conducting polymers there have been demonstrations of vesicle fusion on PEDOT:PSS (31) and polypyrrole (32). What governs the process of vesicle adsorption and rupture is not fully understood, but the first requirement is a hydrophilic substrate. To promote vesicle fusion on PEDOT:PSS an oxygen plasma treatment can be done to introduce $\mathrm{OH}$-groups at the surface, which renders the surface more hydrophilic $(33,34)$.

a

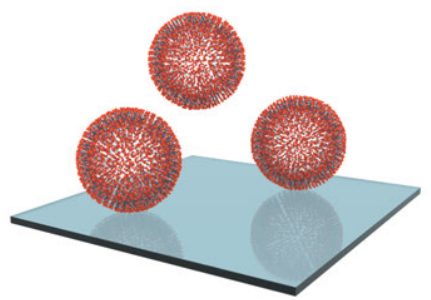

b

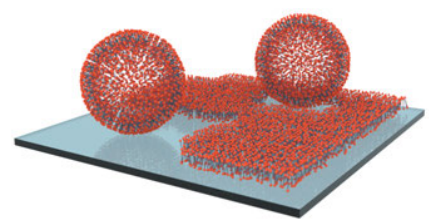

Figure 2.6 Illustration of vesicle fusion. a) Vesicles in suspension adsorb on the substrate. b) The adsorbed vesicles rupture to form a supported lipid bilayer. 
There are two techniques for monitoring the formation of an SLB through vesicle fusion applied in Paper II. One is quartz crystal microbalance with dissipation monitoring (QCM-D), described further in Chapter 5 . The other is a fluorescence microscopy technique based on total internal reflection. When imaging with total internal reflection fluorescence microscopy (TIRF), only the fluorophores closest to the substrate surface contribute to the image (35). Vesicles landing on the substrate appear as high intensity spots in the image. As more vesicles adsorb to the surface the number of spots increases until the vesicles reach a critical surface concentration and start rupturing. The high intensity spots disappear and are replaced with a low intensity sheet covering the surface. This sheet is the supported lipid bilayer. Vesicle fusion has been the technique employed for the formation of bilayers used in Papers II-III.

\subsubsection{Solvent assisted lipid bilayer formation}

The solvent assisted lipid bilayer formation technique (SALB) can be used to form lipid bilayers on metal substrates (36). The lipids are introduced in a flow cell together with an organic solvent, which is then gradually replaced by an aqueous solution. When the exchange is completed a lipid bilayer has formed on the sample surface. As the two liquids mix and the relative composition changes the lipids will undergo a series of phase transitions. Lipid type, temperature and solvent determine what phases will be present in the system throughout the exchange (37). At low water contents the lipids form aggregates exposing the hydrophobic tails to the solvent. Examples of these structures are inverted micelles and multilayer stacks. At high water content the lipids mainly form bilayers that can be present either as vesicles or supported bilayers on a surface. Between these two extremes there are other possible phases, such as monomeric lipids and micelles.

\subsubsection{Langmuir-Blodgett and Langmuir-Schäfer}

Irving Langmuir and Katharine Burr Blodgett developed the Langmuir-Blodgett technique for deposition of monolayers, and stacks thereof on a substrate. The method can be used to deposit amphiphilic molecules, which are molecules that have one hydrophobic and one hydrophilic end. For deposition of SLBs a hydrophilic substrate is repeatedly brought through a lipid monolayer formed at an air-water interface. The monolayer at the interface is referred to as a Langmuir film. Lipids in a Langmuir film are arranged with the hydrophilic heads on the water side and the hydrophobic tails on top, facing the air. This configuration reduces the total interfacial energy in the system (38). A lipid bilayer is produced by emersion of the substrate from the water phase, followed by immersion back into it. The sequence is illustrated in Figure 2.7. During the first emersion step a monolayer with the hydrophilic heads facing the substrate is formed. The 
resulting surface is hydrophobic and upon re-immersion a second monolayer will deposit on top of the first, with the hydrophobic tails facing those of the initial layer, together forming a lipid bilayer. The procedure can be repeated to create stacks of multiple layers (39).
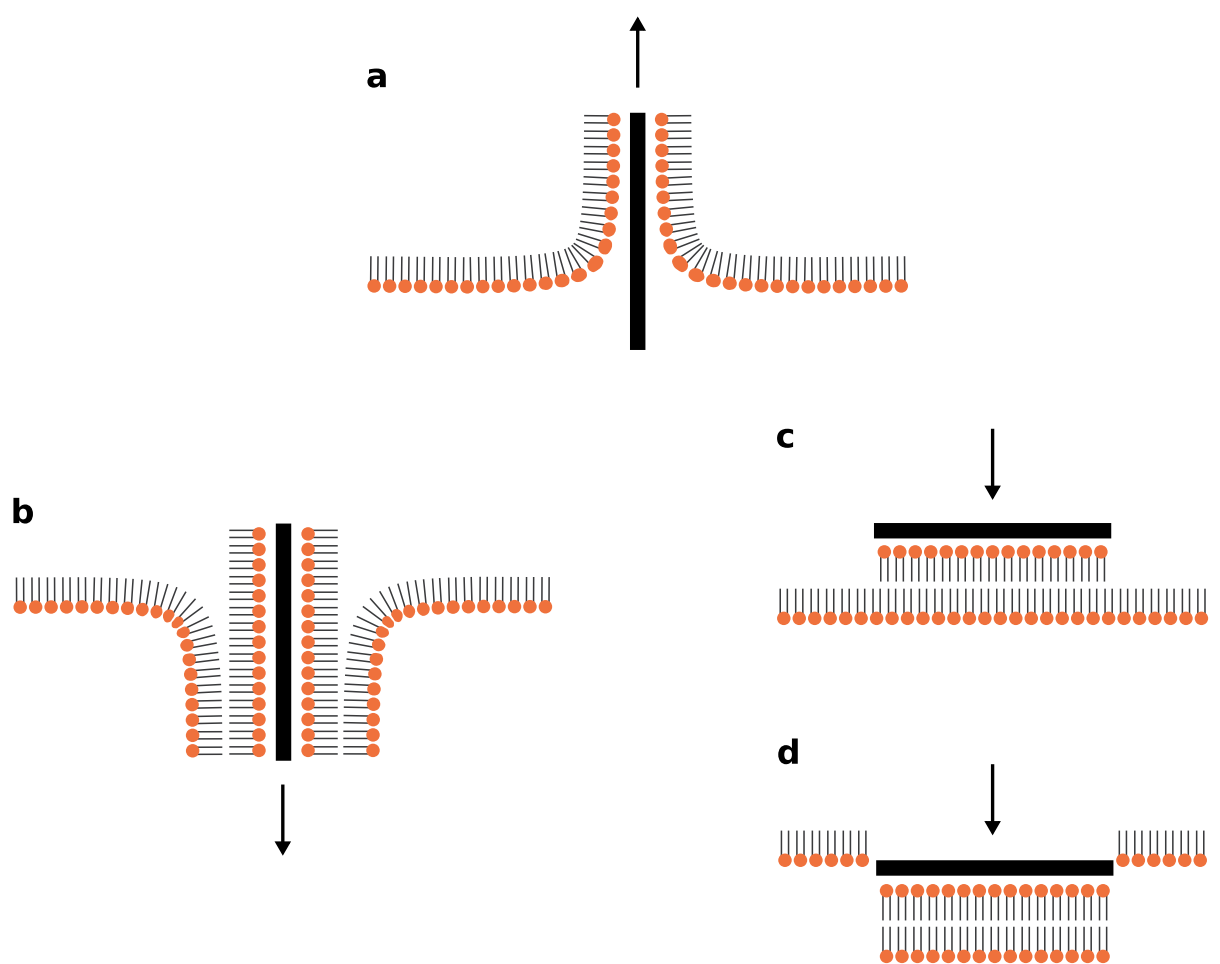

Figure 2.7 Langmuir-Blodgett and Langmuir Schaefer deposition of SLB. The two techniques start with the same step, emersion from the aquatic phase (a). LangmuirBlodgett deposition continues with submersion back through the Langmuir monolayer (b). The continuation of Langmuir-Schaefer is shown in parts $\mathrm{c}$ and $\mathrm{d}$. For these steps the substrate is turned horizontally and brought through the monolayer.

In a Langmuir trough the Langmuir film is compressed from the sides. A Wilhelmy plate monitors the surface pressure to ensure a dense monolayer and proper coverage of the substrate. Surface pressure and pulling rate are the two main parameters influencing the quality of the resulting lipid bilayer on the 
substrate. Other parameters influencing the deposition are $\mathrm{pH}$, temperature, lipid type, substrate, electrolytes.

The Langmuir-Schaefer technique is a variant of the Langmuir-Blodgett that also makes use of the Langmuir trough. During this procedure the first monolayer is formed by emersion of the substrate through the interface, following the procedure of Langmuir-Blodgett deposition. For the second monolayer the substrate is reoriented and brought in contact with the Langmuir film horizontally, allowing the hydrophobic ends of the two molecular layers to interact (4O). Complete immersion of the substrate into the subphase completes the process (Figure $2.7 \mathrm{c}$-d). The Langmuir-Schaefer technique is especially useful for bilayers where the interactions between the polar heads and substrate are weak, as it does not rely on emersion through the air-water interface. 


\section{Materials and properties}

The materials used in the work presented in this thesis were chosen from the toolbox of organic electronics. The term organic refers to materials based on hydrocarbons and includes everything from small molecules such as methane and ethanol to polymers, commonly referred to as plastics. In our everyday electronic equipment, inorganic materials like metals, and especially the semiconductor silicon provide functionality. Still, there are examples of organic electronic components being sold commercially today, with the perhaps most prominent example being the OLED screens based on organic light emitting diodes. The work in this thesis is centered around the polymer poly(3,4ethylenedioxythiophene) (PEDOT) and the ferroelectric polymer polyvinylidene fluoride (PVDF). This chapter will provide a brief introduction to these materials and their properties.

\subsection{Polymers}

A polymer is a large molecule in which the same repeating unit, the monomer, occurs multiple times (41). The words stem from the Greek words poly, mono, and mere, meaning many, one, and part. Figure 3.1 shows the relationship between monomers and polymers. Polymers occur naturally, cellulose and silk are two familiar examples. Since the first synthesis of Bakelite in the early $20^{\text {th }}$ century, synthetic polymers have also become important components of a wide variety of consumer products, from textiles to food packaging (42).

Monomer

Polymer
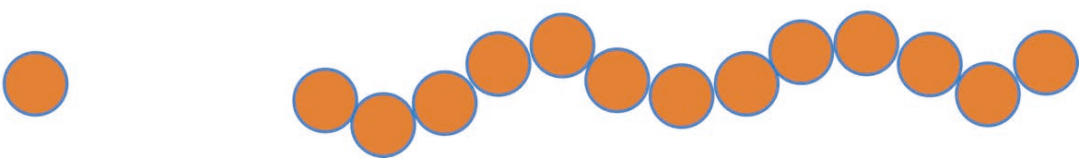

Figure 3.1 Combining monomers results in a polymer.

\subsection{Ferroelectric polymers}

One type of polymers used for organic electronics is the ferroelectric polymers. Ferroelectric materials exhibit a spontaneous electrical polarization that can change direction in response to an external electric field. The term is inspired by the magnetic analogue - ferromagnetic, which is in turn named after the magnetic dipoles present in iron (43). 
a

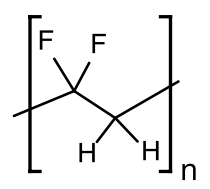

b

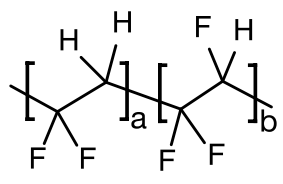

Figure 3.2 Repeating units of the ferroelectric polymers a) PVDF and b) P(VDF-TrFE).

In Paper I the ferroelectric block-copolymer poly(vinylideneflouride-cotrifluoroethylene), $\mathrm{P}(\mathrm{VDF}-\mathrm{TrFE})$, has been used. It is a polymer where segments of the two polymers PVDF and PTrFE occur on the same polymer chain. The chemical structure is shown in Figure 3.2. In the PVDF segments there are electric dipoles, stemming from the high electronegativity of fluoride (44). The PTrFE is not ferroelectric but contributes to the packing of the polymer, giving the films a smoother surface and smaller grain size. Typical morphologies of PVDF and P(VDF-TrFE) are shown in Figure 3.3.
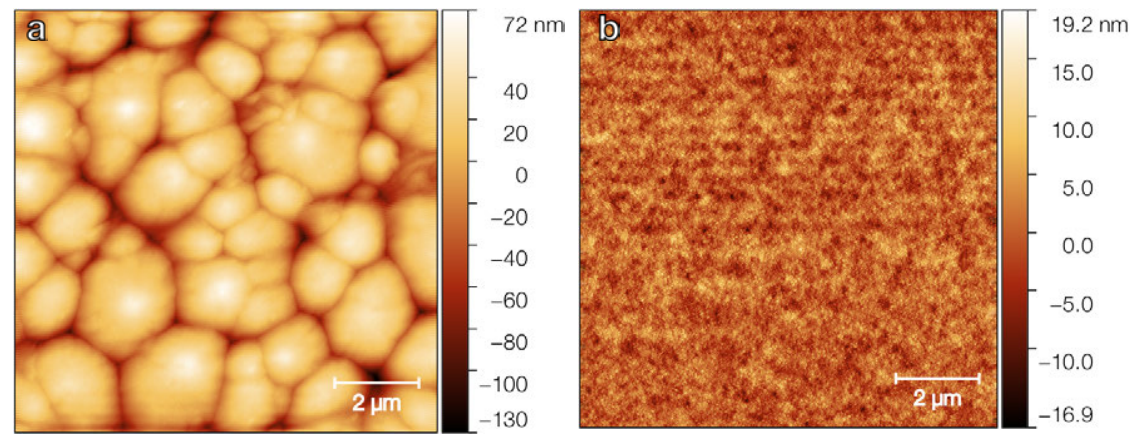

Figure 3.3 Atomic force microscopy topography images of a) PVDF and b) P(VDF-TrFE). Both scans are $10 \times 10 \mu \mathrm{m}^{2}$.

When a $\mathrm{P}(\mathrm{VDF}-\mathrm{TrFE})$ film is first made, the orientations of the dipoles are random. Upon application of an external electric field, the dipoles rearrange and adopt a uniform orientation. The dipoles retain this configuration until an electric field with the opposite sign is applied, causing the dipoles to flip to the opposite orientation. Each ferroelectric material has a minimum electric field, the coercive field, required to reorient its dipoles. For P(VDF-TrFE) the coercive field is $500 \mathrm{kV} / \mathrm{cm}$ (45). Since the field is measured across the polymer, a thicker film requires a larger potential to polarize than a thinner. 
a

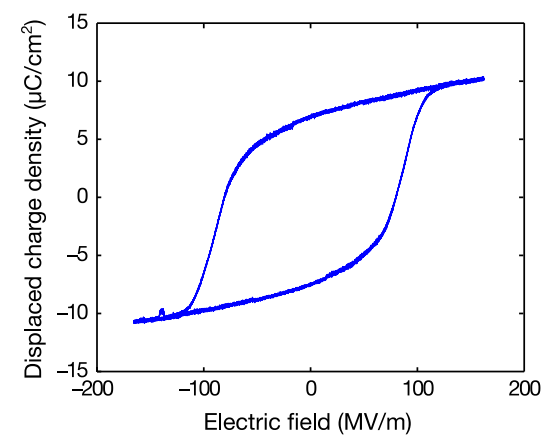

b

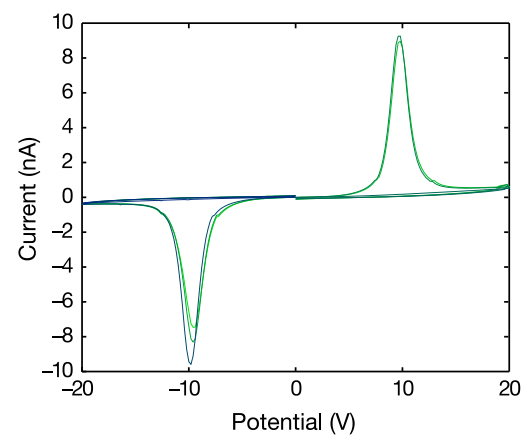

Figure 3.4 a) Charge displacement versus electric field for a P(VDF-TrFE) film sandwiched between metal electrodes. Recorded at $100 \mathrm{~Hz}$. b) Current versus potential for the same film.

A diagram often used to demonstrate the behavior of a ferroelectric material is displaced charge versus electric field, as shown in Figure 3.4a. During the recording a triangular potential signal (with respect to time) is applied to the ferroelectric, covering both positive and negative potentials large enough to flip the dipoles. The graph, often referred to as the ferroelectric hysteresis loop, shows that the charge remains relatively constant until the coercive field is reached and the charge takes on a new value. This new value is then constant until the coercive field, with opposite sign, is reached and the charge is reversed. An alternative way to show the ferroelectric behavior is current versus applied potential. There is a current passing through the circuit just as the dipoles flip and the charge takes on its new value. Sweeping to both negative and positive potentials then gives two current peaks, as shown in Figure 3.4b. Since the ferroelectric materials retain their polarization also in the absence of an electric field they have applications within memory technology (46).

\subsection{Conjugated polymers}

Conjugated polymers have alternating single and double bonds along their backbones. These polymers are able to conduct electricity and the origin of this ability is found in the electronic structure of the bonds formed between the atoms (47). The carbon atoms along the polymer chain form covalent bonds with each other, meaning they come together and share electrons. The electrons move around the nuclei and are likely to be found within a certain volume of space near the nuclei. These volumes are called orbitals and can be thought of as electron clouds. Atoms have atomic orbitals that combine to form molecular orbitals when 
the bonds are formed (48). Carbon atoms have four electrons available to form bonds with other atoms. To complicate the picture, the original $s$ and $p$ atomic orbitals in carbon can be combined to form hybrid atomic orbitals, see Figure 3.5 .

a

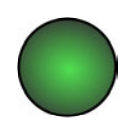

b

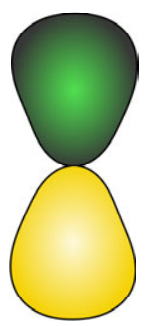

c

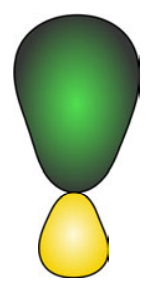

Figure 3.5 Schematics of atomic orbitals. a) $s$-orbital b) $p$-orbital c) hybrid $s p^{3}$-orbital.

In the case of the small molecule methane $\left(\mathrm{CH}_{4}\right)$, the carbon atom in the center of the molecule forms four molecular orbitals together with atomic orbitals from the hydrogen atoms. Molecular orbitals like these, formed between hybrid orbitals, or between hybrid orbitals and $s$-orbitals are called $\sigma$-orbitals, and the bonds are $\sigma$-bonds. The electrons in the hybrid orbitals repel each other, giving the methane molecule its tetrahedron shape, with the hydrogen atoms in the four corners. More carbon atoms can be added to replace one or more of the hydrogen atoms. These carbon atoms will also each contribute one electron to every binding and strive to place themselves in the center of their own tetrahedrons. The bindings formed in this way are what we call single bonds, but carbon atoms are also able to form double and triple bonds with more electrons from each carbon atom participating in the bonds.

In order to form a double bond, the carbon atom forms three hybrid orbitals, each containing one electron. These orbitals are oriented in a plane, with $120^{\circ}$ angles between them. Perpendicular to this plane is a fourth orbital, which is not a hybrid orbital, but a standard $p$-orbital. To make the double bond, one of the hybrid orbitals on each atom align and come together and form a $\sigma$-orbital, while the $p$-orbitals are positioned parallel to each other. Even though the atomic orbitals are mainly reaching outwards from the plane, there is still overlap, as illustrated in Figure 3.6. In a hydrocarbon chain with mainly single bonds and one double bond two of the atoms will display the hybridization just discussed, with three hybrid orbitals and one $p$-orbital. This hybridization is called $s p^{2}$, from the atomic orbitals needed to construct the hybrid orbitals: one $s$-orbital and two $p$-orbitals, making three molecular orbitals in total. The remaining carbon atoms will be $s p$-hybridized, meaning they use one more $p$-orbital to form the hybrid orbitals. The double bond is found between the two $s p^{2}$-hybridized atoms. 


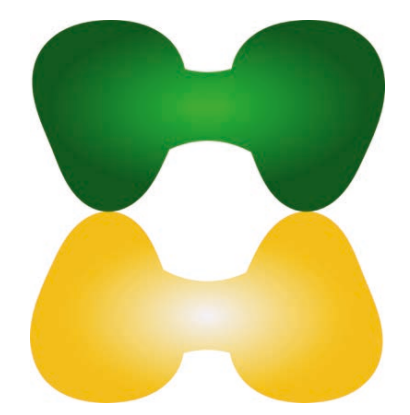

Figure $3.6 \pi$-orbital and $\pi$-binding formed from $p$-orbitals on two carbon atoms. Between the atoms there is also a $\sigma$-bond that has been left out of this illustration.

In a conjugated system, all carbon atoms are $s p^{2}$-hybridized. One example of such a molecule is benzene. Benzene has six carbon atoms and alternating single and double bonds. One way to draw benzene is shown in Figure 3.7a, with the single and double bonds clearly marked. Figure $3.7 \mathrm{~b}$ shows a different representation, where the double bonds are instead illustrated as a ring shared among all atoms. This interpretation of the structure is thought to better reflect how the electrons and double bonds in the conjugated system behave, shifting between all positions rather than being fixed to certain atoms. The same effect is thought to occur also in linear molecules and polymers, making the electrons in the $\pi$-bonds delocalized along the conjugated chain. Once a charge is introduced, by adding or removing an electron, this charge can use the delocalization to move from one end of the chain to the other. The delocalization of electrons in the conjugated system is the origin of its ability to conduct electricity.
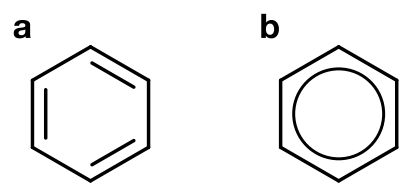

Figure 3.7 Benzene drawn in two ways. a) Single and double bonds drawn in fixed positions. b) Conjugation illustrated by a ring in the center of the molecule.

\subsubsection{PEDOT:PSS}

The conducting polymer of choice for papers II-IV was poly(3,4ethylenedioxythiophene) (PEDOT) doped (see Section 3.3.3) with the polyanion polystyrene sulfonate (PSS). The chemical structures are shown in Figure 3.8. 
PEDOT is a member of the polythiophene family and was developed in the 1980s to address challenges with stability experienced with the already used polymers polyacetylene and polypyrrole (49). The early applications of PEDOT included antistatic coatings and high-frequency applications in capacitors.

a

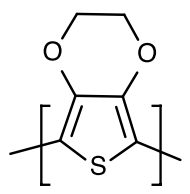

b<smiles>CC(CC(C)(C)C)c1ccc([S+](=O)(=O)[O-])cc1</smiles>

Figure 3.8 The repeating units of a) PEDOT b) PSS

\subsubsection{Conductivity and resistivity}

A material's conductivity is a measure of its ability to pass charge carriers. The conductivity, $\sigma$, is governed by the density of charge carriers in the material and their mobilities, giving:

$$
\sigma=n e \mu_{e}+p e \mu_{h}
$$

where $n$ and $p$ are the negative and positive charge carrier concentrations, $e$ the elementary charge, and $\mu_{e}$ and $\mu_{h}$ the charge carrier mobilities for the electrons and holes respectively.

The inverse of the conductivity is the resistivity, $\rho$. Together with the dimensions of conductor, the resistivity determines the resistance by

$$
R=\rho \frac{L}{A}
$$

where $L$ is the length and $A$ the cross-sectional area.

\subsubsection{Primary doping}

Addition of charge carriers to a semiconductor is referred to as doping. In conjugated polymer chains, adding or removing an electron will add charge carriers and increase conductivity. Polymers where doping is achieved through addition of electrons are said to be n-type polymers, meaning they conduct negative charge carriers. In p-type polymers, on the other hand, the charge carrier is positive. The positive charge carrier is actually a missing electron - a hole. There are two main strategies to dope a conjugated polymer: chemically and electrochemically. In chemical doping a substance is added that will either donate electrons to the polymer chain or take electrons from it (5O). A polymer gaining 
electrons is reduced and the chemical added is called reductant. Similarly, an oxidant will oxidize the polymer by taking electrons from it. For electrochemical doping an applied potential introduces or removes electrons from the polymer chain. Electrochemical doping requires the polymer to be in contact with, and exchange, mobile ions to maintain charge neutrality in the material. The compensating ions remain inside the polymer film while the doping level is maintained. Similar compensating elements can also be introduced during the polymerization of the polymer, as is the case for PEDOT oxidatively polymerized in a water solution of PSS (51). The resulting polymer is in its doped state, and PSS serves as a stabilizing dopant. The electrochemical transistor described in the next chapter is a device based on electrochemical doping and de-doping. The ion exchange, and the related ion migration, during doping and de-doping of the polymer can cause the polymer to swell and shrink $(52,53)$. This feature can be harnessed to create actuators used for microscopic machines and is investigated for possible applications within synthetic muscles $(54,55)$. In the work within this thesis the volumetric changes were mainly a cause of concern about the stability of the top coatings applied in Paper II. Problems with cracks forming during the swelling of the underlying PEDOT:PSS were resolved by decreasing the thickness of the top coatings.

\subsubsection{Secondary doping}

PEDOT:PSS is commercially available as a suspension in water, intended to simplify fabrication processes. The suspensions are compatible with many of the standard film making techniques, such as spin-coating and printing. However, the conductivities of the resulting films are low if the suspensions are used as received. The reason is thought to be found in the morphology of the film, where the conducting PEDOT resides in isolated islands embedded in PSS, impeding charge transport throughout the film (56). A higher overall conductivity is achieved when connectivity between PEDOT clusters is improved. There are multiple ways to perform conductivity enhancing treatments (57). One of them, which is used in this thesis, is to add a high boiling point solvent to the aqueous suspension before film formation (58). High boiling point solvents used for this purpose are for example dimethyl sulfoxide (DMSO) and ethylene glycol (EG). Since these additives increase the conductivity in the film without directly increasing the number of charge carriers they are referred to as secondary dopants, to separate them from the primary dopants that introduce charge carriers (59). On a film level, the addition of the high boiling point solvent increases the charge carrier mobility, thereby increasing overall conductivity of the material. 


\subsection{Double layer capacitance}

All papers included in this thesis contain measurements in aqueous environments. It is therefore relevant to consider how charge can be stored at the interface between an electrode and electrolyte. The concept of the electrical double layer (EDL) was first introduced in the 1850 os by Hermann von Helmholtz $(60)$. Over the years the understanding of the EDL has grown and several subsequent models have been proposed to capture the gradual potential drop in the solvent and the motion and adsorption of ions. The initial thoughts of Helmholtz are, however, sufficient to understand the working principles of the devices in this thesis.

At the surface of the electrode, there is a layer of charges, attracting ions of the opposite polarity from the solution. Together, these two layers of charges form a double layer. Each layer can be considered to act as an electrode in a parallel plate capacitor. The ions are surrounded by a solvation shell of molecules from the solvent, which together with the ionic radius defines the distance, $d$, between the charges. For a parallel plate capacitor, the capacitance, $C$, is:

$$
C=\varepsilon \frac{A}{d}
$$

where $\varepsilon$ is the permittivity of the dielectric, in this case the solvent, and $A$ the electrode area. Since the distance between the charges is small, the double layer capacitance can be high. A common way to increase the capacitance further is to use porous electrodes and thereby increase the active surface area. In the case of PEDOT:PSS, the double layer is thought to extend throughout the bulk $(61,62)$. For this reason it can be more relevant to define a volumetric capacitance, rather than the areal double layer capacitance used for metal electrodes (63). 


\section{Organic electrochemical transistors}

\subsection{Introduction to transistors}

A transistor is a device where the current flowing between two electrodes is modulated by a third electrode. The first transistor was demonstrated at Bell labs in 1947 and has since become a key component in electronic equipment (64). There are two sets of terminologies to describe the components of the transistor, one for bipolar junction transistors, and one for field-effect transistors. The transistor developed at Bell labs falls in the former category, and its contacts are named emitter, collector and base (65). Field-effect transistors instead have the corresponding contacts source, drain and gate. For both transistor categories, a small potential or current signal at the base/gate results in a large change in current between emitter/source and collector/drain. Once the transistor was invented it quickly replaced the preceding vacuum tube technology and enabled the digital revolution (66). The main benefits over the vacuum tube were the miniaturization, reliable operation and lower power demands of the transistors.

The organic electrochemical transistor (OECT) shows more functional resemblance with the field-effect transistors and source, drain and gate are thus used to describe its electrodes. The first OECT was demonstrated in 1984, although at the time the authors referred to it as a chemiresistor (67). The active layer of the device was electropolymerized across three gold electrodes, later acting as source, drain and gate. With the gate electrode between the other two electrodes, underneath the polypyrrole film, a potentiostat was used to regulate the oxidation state of the polymer and its conductivity, producing typical transistor behavior.

\subsection{OECT structures}

As mentioned in the previous section, the OECT has three electrodes: source, drain and gate. Since the first demonstration of the OECT, the gate has now moved away from the channel and is typically not in direct contact with it. The channel is made of an organic semiconductor, being contacted at either end by source and drain electrodes, and is allowed exchange of ions with a contacting electrolyte. This exchange of ions is essential to the modulation of doping level in the channel, and thereby the regulation of the current passing through the channel. The most common form of OECT transistor channel is a thin polymer film deposited on a flat substrate, but there are also examples where the channel has been coated on a wire (68), in a gel (69), or even inside a plant (70). In this 
thesis, the focus is on thin-film channels on planar substrates. The gate electrode is in contact with the same electrolyte as the channel. Common gate options are non-polarizable electrodes such as $\mathrm{Ag} / \mathrm{AgCl}$, metals and conducting polymers. Transistors fabricated on planar substrates can have the gate on the same substrate next to the channel (Figure 4.1a), or have the gate brought in contact with the electrolyte from the top (Figure 4.1b). The planar design was developed as a printable device utilizing PEDOT:PSS ink, eliminating the need for electropolymerization of the channel material (71).

Another important part of the OECT design is the encapsulation which ensures that only the channel and gate are in contact with the electrolyte, keeping connectors and conductors dry and preventing electrochemical reactions.

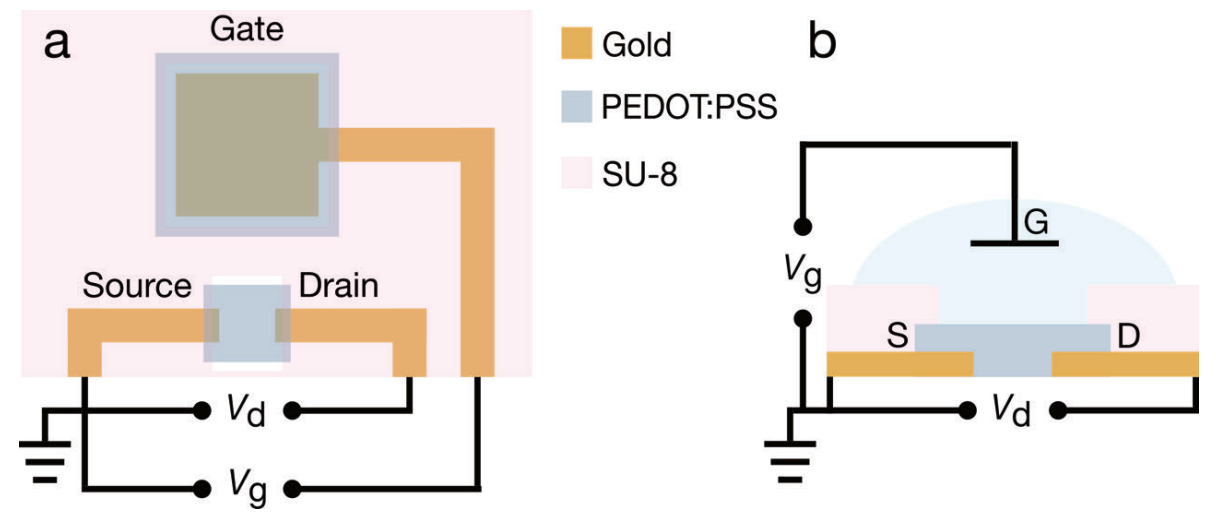

Figure 4.1 a) Top view of a planar PEDOT:PSS OECT with the gate next to the channel. In this case the gate is coated with PEDOT:PSS, and SU-8 provides the encapsulation. The liquid electrolyte has been left out for clarity, but covers both gate and channel. b) Crosssectional view of a top-gated OECT, in which the gate electrode is brought in contact with the electrolyte from the top.

\subsection{Function}

Since the transistors used for the experiments in this thesis had PEDOT:PSS channels, the description of how the OECT works will be centered around p-type depletion mode transistors. P-type refers to the channel being a hole conductor, meaning the charge carriers are positively charged, as opposed to electrons that are negatively charged and the main charge carrier type in n-type materials. In depletion mode transistors, the channel is natively in its most conductive state and the applied gate potential pushes positive ions into the channel. As more positive ions are added to the channel, the holes leaving the channel at the drain 
and are not replaced by incoming holes from the source, resulting in fewer charge carriers on the polymer chains. A reduction in charge carrier concentration lowers the electrical conductivity in the channel and thereby increases the channel resistance. With a higher channel resistance, the drain current is lowered. The de-doping process is depicted in Figure 4.2.

a

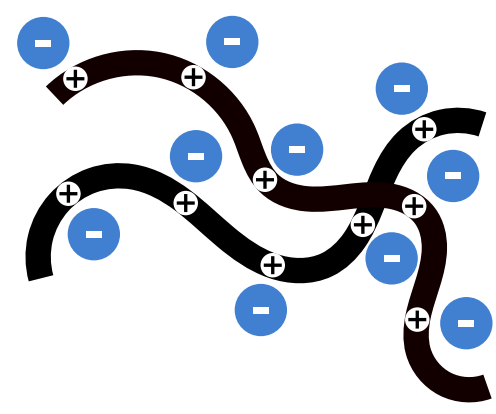

b

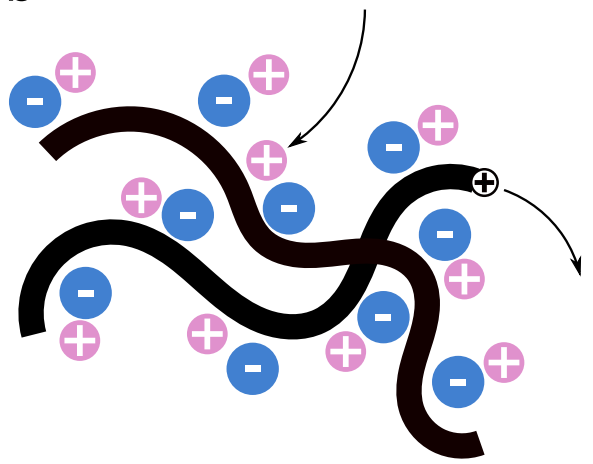

Figure 4.2 De-doping of PEDOT:PSS in a depletion mode OECT. a) The native state with the positive charges on the polymer chains being compensated by negative ions. b) As a positive gate potential is applied, positive ions enter the film and the charge carriers leave the chains at the drain contact.

Since gate and channel are only connected through the ions present in the electrolyte, and the current measured through the channel is electronic, it can be useful to conceptually divide the OECT into two separate circuits. There is an ionic circuit between gate and channel, and an electronic circuit along the channel, as illustrated in figure 4.3. The elements present in the ionic circuit depend partly on the gate, but the solution resistance, $R_{\mathrm{S}}$, and the double layer capacitance, $C_{\mathrm{ch}}$, at the interface between channel and electrolyte are always present. In the planar device shown in figure 4.3 there is also a double layer capacitance at the gate, $C_{g}$. The electronic circuit is represented by a variable resistor, $R_{\mathrm{ch}}$. The charge accumulated on the channel capacitor determines the de-doping in the channel thereby turning the dial on the variable resistor in the electronic circuit (11). 


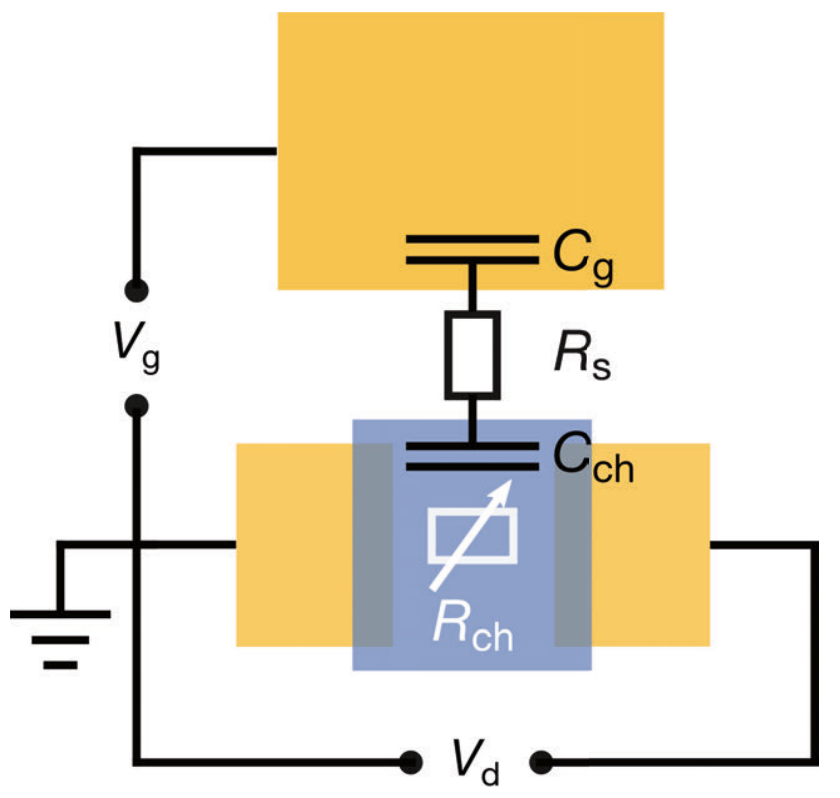

Figure 4.3 Electronic and ionic circuit of a PEDOT:PSS channel OECT with gold gate. The ionic circuit elements present between gate and channel are drawn in black, while the electronic circuit is shown in white.

\subsection{Characteristics}

Two sets of curves are typically used to demonstrate the behavior of OECTs. One is the output characteristics, where the drain current is recorded while sweeping the drain potential. The procedure is repeated for several gate potential values. An example of output characteristics is shown in Figure 4.4a. The other common curve is the transfer curve. Here, the drain current is shown versus gate potential. The drain potential is kept at a constant value. Transfer curves show how well the transistor translates an altered gate potential into changes in drain current. From the transfer curve, the transconductance, $g_{\mathrm{m}}$, for the transistor can be determined. The transconductance is found from the slope of the transfer curve, $g_{\mathrm{m}}=\partial I_{\mathrm{d}} / \partial V_{\mathrm{g}}$, and is a measure of how much the current changes with the applied gate potential. Figure $4.4 \mathrm{~b}$ shows a transfer curve, and the corresponding transconductance. 

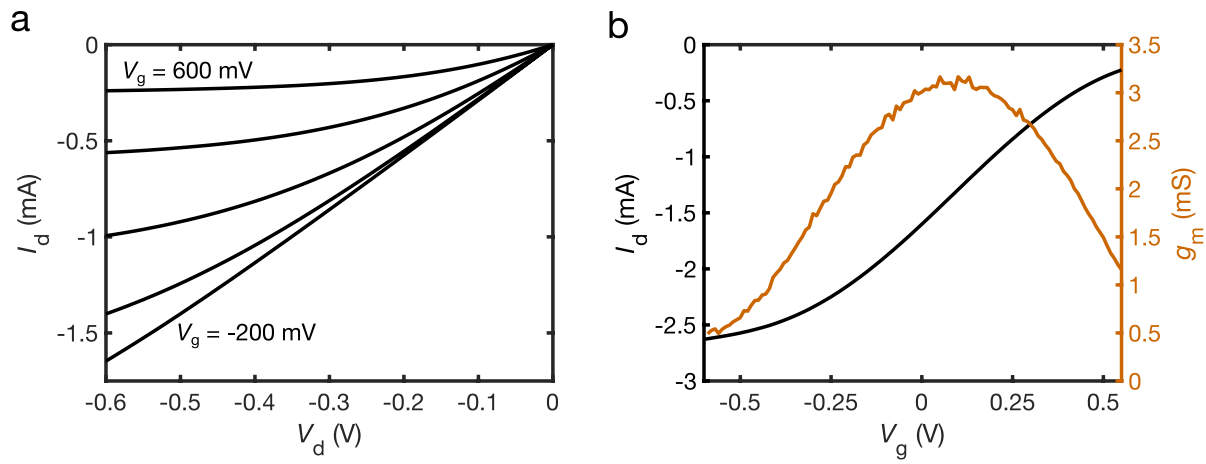

Figure 4.4 a) Output characteristics for a PEDOT:PSS OECT with PEDOT-coated gate. Gate potentials range from $-200 \mathrm{mV}$ to $600 \mathrm{mV}$ in $200 \mathrm{mV}$ steps. b) Transfer curve recorded at $-800 \mathrm{mV}$ drain potential. The transconductance is shown on the right side $y$-axis. The data is a selection from a bigger set used in Paper IV.

Since the OECT is dependent on ion movement to occur before changes in gate potential can influence the drain current, OECTs are considered slow devices. The capacitive and resistive circuit elements indicated in Figure 4.3 together determine the speed of the transistor. Generally, a small transistor channel is faster than a larger one, and keeping the solution resistance down by placing the gate close to the channel can also increase the speed. The transistors used in this thesis could typically keep up with small gate signals of a few hundred Hz. When higher frequency signals are applied, the response in the gate current becomes smaller and smaller. There is also a phase shift in the gate current, meaning that peak channel resistance does not occur simultaneously with the peak gate potential. An example of an OECT frequency response is shown in figure 4.5.

a

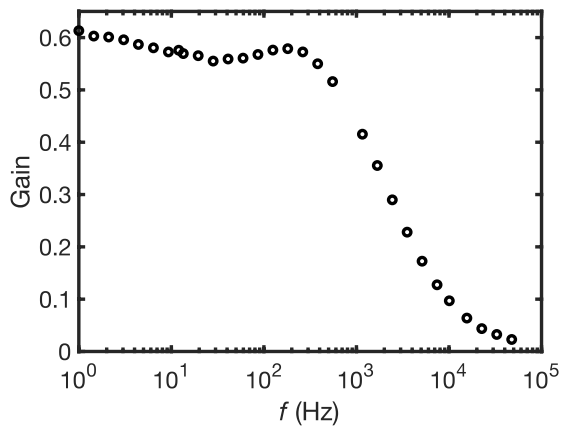

b

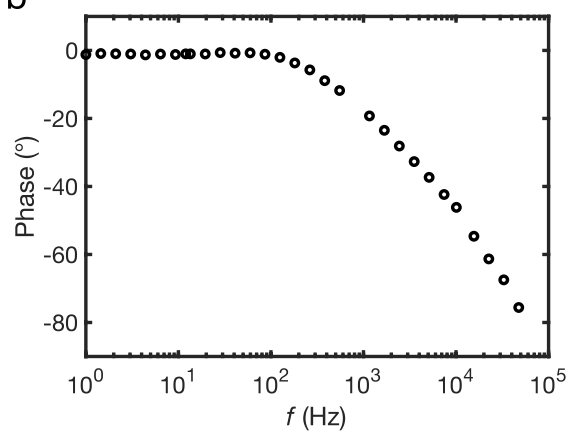

Figure 4.5 Frequency response of an OECT recorded at $V_{d}=-370 \mathrm{mV}$ using an amplifier circuit. a) Gain, scaling with transconductance b) Phase angle. The channel material was PEDOT:PSS and the gate was gold. The data also appears in Paper III. 


\subsection{OECT models}

The development of OECT models has moved forward along with the increasing knowledge about conjugated polymers (72). In this thesis the model proposed by Bernards and Malliaras in 2007 has been the foundation for Papers III and IV (73). In paper III, the Bernards model was combined with a simplified version of the equivalent circuit model proposed by Faria and Duong $(74,75)$. This section will mainly describe these two models, but a short description of more complex models will also be included.

\subsubsection{Bernards and Malliaras 2007}

The Bernards model links the charge accumulated locally in the channel to its degree of de-doping and channel conductivity modulation. The circuit used to construct the model is shown in Figure 4.6.
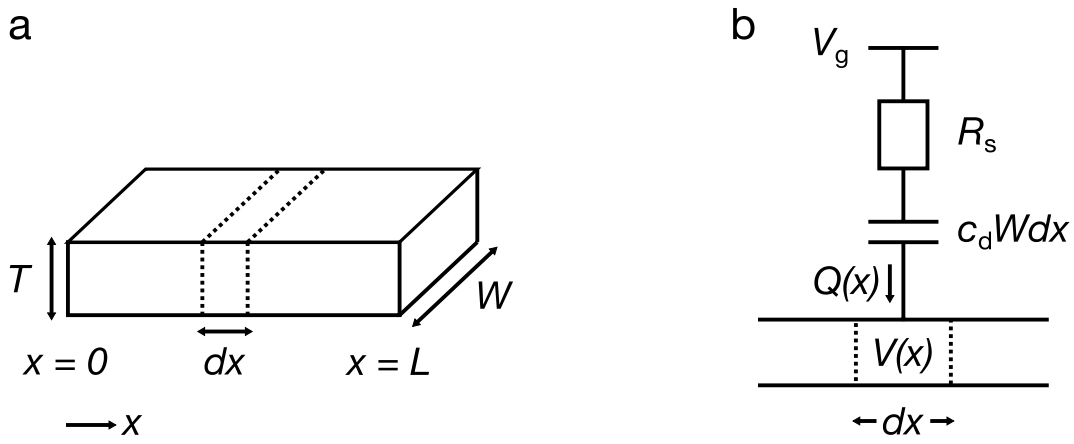

Figure 4.6 a) Channel geometry and b) Circuit diagram used to construct the Bernards model. The double layer capacitance, $c_{d}$, refers to the measured areal capacitance for the channel. The charge accumulated in a portion of the channel, $d x$, is coupled to the local channel potential, $V(x)$.

For steady-state operation, three operational regimes were identified, each with its own drain current equation. One of the regimes only concerns operation at positive drain potentials and will not be described here, since that biasing is not commonly used for PEDOT:PSS OECTs and has not been applied in this thesis. The two regimes observed at negative drain potentials are one with some level of de-doping along the entire channel length, and one in which a region closest to the drain has been completely depleted of charge carriers. The depletion causes the drain current to saturate and it remains at the saturation level also if the drain potential is decreased further. Saturation occurs when $V_{\mathrm{g}}-V_{\mathrm{d}} \geq V_{\mathrm{p}}$. The pinch-off potential, $V_{\mathrm{p}}$, is the drain potential at which the drain current saturates at 
$V_{\mathrm{g}}=\mathrm{o} \mathrm{V}$. The drain potential at saturation is given by $V_{\mathrm{d}}^{\mathrm{sat}}=V_{\mathrm{g}}-V_{\mathrm{p}}$. At saturation, the drain current is given by

$$
I_{\mathrm{d}}=-\frac{G V_{\mathrm{d}}^{\mathrm{sat}^{2}}}{2 V_{\mathrm{p}}}
$$

where $G$ is the initial conductance in the channel. Since $V_{\mathrm{d}}^{\text {sat }}$ varies with gate potential, the transconductance is predicted to decrease linearly with $V_{\mathrm{g}}$ in this regime.

At smaller potential differences between drain and gate there is a regime where the charge carrier concentration is modulated along the entire channel. In this regime the transconductance does not vary with gate potential. The drain current in this regime is

$$
I_{\mathrm{d}}=G\left(1-\frac{V_{\mathrm{g}}-1 / 2 V_{\mathrm{d}}}{V_{\mathrm{p}}}\right) V_{\mathrm{d}}
$$

In practice, the transconductance often displays more of a peak than a section of a constant value (see example in figure 4.4b). Later additions to the Bernards model have strived to capture this behavior by considering variations in charge carrier mobility with concentration (76). Also, modifications to capture accumulation-type OECTs have been proposed (72). In Paper IV, the application of the Bernards model was expanded into negative gate potentials identifying three additional operational regimes.

In addition to equations for steady-state drain currents, the Bernards model also provides an expression for the transient drain current response to a gate potential step:

$$
I_{\mathrm{d}}\left(t, V_{\mathrm{g}}\right)=I_{\mathrm{ss}}\left(V_{\mathrm{g}}\right)+\Delta I_{\mathrm{ss}}\left(1-f \frac{\tau_{\mathrm{e}}}{\tau_{\mathrm{i}}}\right) e^{-t / \tau_{\mathrm{i}}}
$$

where $I_{\mathrm{ss}}$ is the steady-state drain current, and $\Delta I_{\mathrm{ss}}=I_{\mathrm{ss}}\left(V_{\mathrm{g}}\right)-I_{\mathrm{ss}}\left(V_{\mathrm{g}}=0\right)$. The proportionality constant, $f$, accounts for de-doping non-uniformity along the channel and is in the range $0 \leq f \leq 1 / 2$. Later models have provided methods for determining $f(74)$. The time constants, $\tau_{\mathrm{e}}$ and $\tau_{\mathrm{i}}$ are the electronic and ionic transit times, respectively and $t$ is the time counting from the step.

\subsubsection{Equivalent circuit models}

Another approach to model OECTs is to assign an equivalent circuit to capture transistor behavior. These models have successfully been able to predict transient drain currents in OECTs, and have been extended to describe impedance biosensing (75). The equivalent circuit describing the transistor is shown in figure 4.7. The channel is represented by a capacitor, $C_{\mathrm{ch}}$, and a resistor, $R_{\mathrm{ch}}$, in parallel. The capacitor represents the double layer capacitance formed at the 
interface between channel and electrolyte. $R_{\mathrm{ch}}$ represents the resistance for charge transfer between electrolyte and channel, often called charge transfer resistance. In series with these elements are an electrolyte resistance, $R_{\mathrm{s}}$.

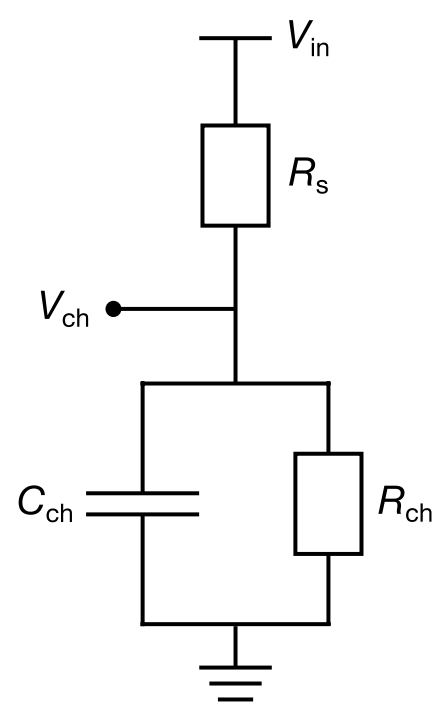

Figure 4.7 Equivalent circuit used to construct the equivalent circuit model proposed by Faria and Duong.

A step in the gate potential results in a capacitive current flowing between gate and channel. This current is divided between the source and drain contacts. For a channel where source and drain are kept at the same potential, the current is divided evenly. If the channel is biased, more current will flow to one of the electrodes. The portion of the gate current going to the drain is incorporated in the transient drain current response, and its size is captured in the proportionality constant $f$, also present in the Bernards model. The other contribution to the transient current is the equilibration of the drain current while the channel is de-doping. This part is proportional to the transconductance and the time-dependent channel potential, $V_{\mathrm{ch}}(t)$. The transient gate current and the channel equilibration current is then added to the drain current before the gate potential step, $I_{0}$, yielding the drain current expression

$$
I_{\mathrm{d}}(t)=I_{0}-f \cdot I_{\mathrm{g}}(t) \pm g_{\mathrm{m}} V_{\mathrm{ch}}(t)
$$


The assumptions that the transient drain current is dependent on the potential drop over the channel, and that equivalent circuits can be used to describe barrier layers added to the transistor were used in Paper III.

\subsubsection{Drift-diffusion models}

Drift-diffusion models for OECTs pay closer attention to the impacts of ion movements on transistor behavior. These models cover the transport of ions between gate and channel, and also consider the mixed electron and ion conduction in the conjugated polymer (77). The more detailed understanding of ion distribution along the channel can then be used to generate a more sophisticated picture of the local potential and conductivity (78). Drift-diffusion models can also be applied in 2D to account for ion transport both in the direction between electrolyte and substrate and between source and drain (79).

\subsection{Sensors based on OECTs}

Due to their capability to transduce ionic to electronic signals and operate in aqueous environments, OECTs have attracted interest for use in biosensors $(80)$. The transistors can be used to quantify a chemical analyte $(81,82)$, study biological barriers $(83,84)$ or record electrical activity in the body $(85)$. At the core of the sensing is a change in the potential drop between channel and electrolyte, influencing the doping level in the channel and thereby also the drain current that is typically used for read-out. Depending on how the potential drop changes, different transistor designs are favorable.

For sensing of electroactive species, the chemical potential in the electrolyte changes, altering the potential drop at the interface between channel and electrolyte. In order to maximize the sensor signal in this sensing mode, the transistor should go from a small potential drop at the channel-electrolyte interface to a large one. A straight-forward way to limit the initial potential drop at the channel-electrolyte interface is to use a transistor with a small gate-tochannel capacitance ratio (86). This way, in the absence of analyte, a large part of the total potential drop falls over the gate-electrolyte interface. Once the analyte is introduced, the potential distribution changes and the potential difference between channel and electrolyte increases, modulating the doping level in the channel and affecting the channel resistance.

For impedance sensing, usually a capacitive layer is added between gate and channel. This added layer will take a part of the total potential drop and thereby reduce the drop at the channel-electrolyte interface. For effective sensing, the added layer should then have a large impedance compared to the channelelectrolyte interface. One way to realize a low impedance channel is to increase 
its capacitance by increasing channel volume. In Paper III, a special case of impedance biosensing is investigated where a capacitive layer is added to the gate. 


\section{Experimental techniques}

This chapter will introduce the most important experimental techniques applied in the work with this thesis. The descriptions focus on the techniques as they have been applied in the papers, and do not necessarily include all other possibilities and options available.

\subsection{Evaporation}

Evaporation is a technique for depositing material onto a substrate. In this work it has been used to create metal layers later patterned to form electrodes, conductors and contacts for devices. The material to be deposited is heated under vacuum, causing some of it to enter the vapor phase and leave its source (87). The vapor is spread away from the source and condensates on colder surfaces. Some of the evaporated material will reach the substrate and deposit there. One way of heating the source material, which is used in our lab, is to place the material in a carrier vessel, called a boat. The boat is resistively heated when a current is passed through it. During the evaporation, the thickness of the deposited film is measured, often using a quartz crystal microbalance that monitors the mass added to the crystal surface. The deposition rate can be controlled by adjusting the current, and thereby the boat temperature.

\subsection{Spin-coating}

Spin-coating has been the go-to technique for producing the polymer films in this thesis. The film material is initially in solution or suspension and is dispensed onto the substrate. The substrate is kept level but is rotated to spread the suspension outwards. At the same time, the solvent evaporates, gradually increasing the viscosity of the solution. The nature of the solvent, angular rotation speed and initial concentration are important factors influencing the film properties (88).

\subsection{Photolithography}

The transistors used in Papers III-IV have been manufactured using photolithography with ultraviolet (UV) light. The technique utilizes a photosensitive polymer, called photoresist, that is sensitive to UV light. For a positive resist, the regions exposed to UV light become more soluble and are washed away in the following developing step. A negative resist becomes less soluble upon exposure, and instead the unexposed areas are removed in the development (89). 
By illuminating the resist through a mask, the pattern of the mask can be transferred onto the resist. The pattern in a positive resist corresponds to the mask pattern, while a negative resist displays an inverted pattern.
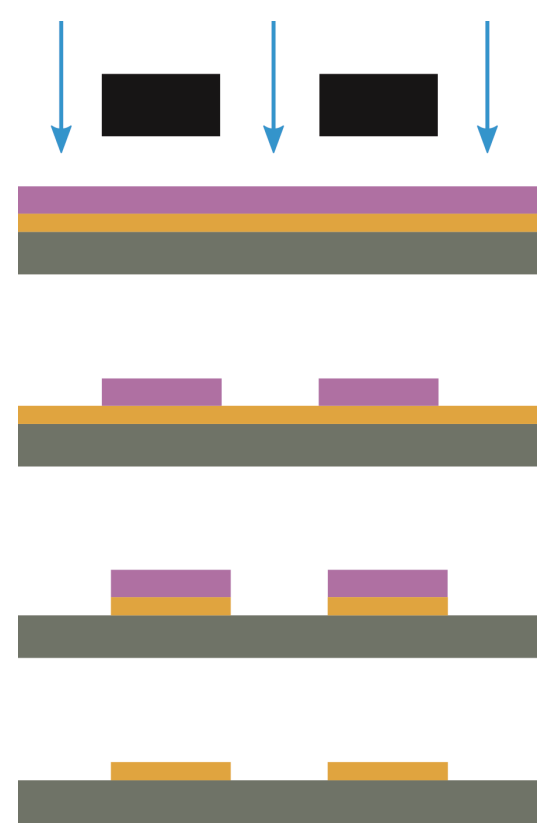

Figure 5.1 Patterning sequence for photolithography using positive photoresist and etching. The sequence starts in the top figure with illumination through the mask, and is followed by developing, etching and finally, stripping of the resist.

The patterning of the transistors has been done using etching. The patterning process is illustrated in figure 5.1. First, the material to be patterned is deposited onto the substrate. A photoresist layer is then added on top of the stack, and subsequently exposed through the mask and developed. The material covered by photoresist is protected, while the rest is removed during an etching step. Once the material is etched the remaining photo resist is removed. Metals were deposited using thermal evaporation, and polymers using spin-coating. Metals were etched in a wet etch process, in which the substrate is immersed in etching solution. The reactant in the etching solution reacts with the exposed metal, forming soluble products that are free to leave the surface. Polymers were etched using reactive ion etching (RIE). RIE is a dry etch technique combining physical bombardment of ions with chemical reactions on the sample surface (90). 


\subsection{Impedance spectroscopy}

To determine the electrical properties of an SLB, impedance spectroscopy is a powerful technique. During the recording of a spectrum the system under examination is subjected to a small alternating potential (91). The frequency of the AC potential is varied from high to low frequencies while the current passing through the system is measured to determine its impedance. Automated measurements can be done using special impedance spectrometers, but many potentiostats are also able to perform impedance spectroscopy. There are several ways to display the data collected during a frequency scan, with the Bode and Nyquist plots being the most popular choices when working with bilayer membranes. In the Bode plot the logarithm of the frequency is plotted on the x-axis. There are then two y-axis curves, one displaying the logarithm of the absolute value of the impedance, and the other the phase angle between the input and output signals. Using the raw data values presented on a log-scale, instead of the logarithms is also common. In the Nyquist plot, for each frequency point, the imaginary part of the impedance is plotted against the real part. Figure 5.2 shows examples of Bode and Nyquist plots.

a

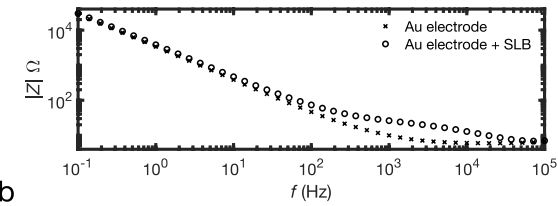

b

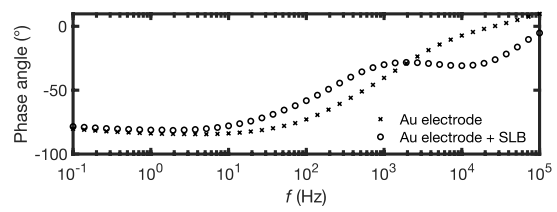

C

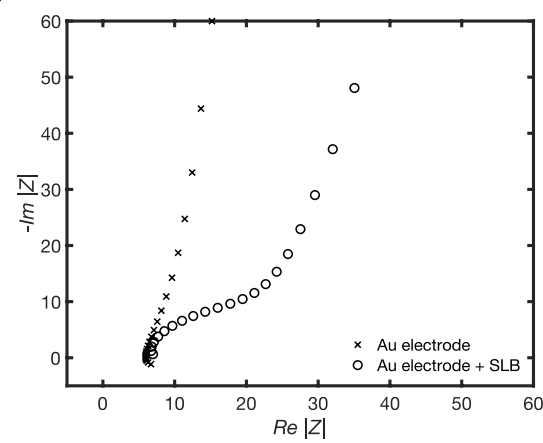

Figure 5.2 Impedance spectra for gold electrode $(x)$ and gold electrode with DOTAP bilayer (o). a) Bode modulus b) Bode phase angle c) Nyquist plot. The data is from Paper III.

Equivalent circuits are needed to analyze and interpret the data. These are electrical circuits with elements that are tied to various properties of the system under study. Ideal elements that can be found in typical equivalent circuits are resistors and capacitors. The impedance of the resistor is frequency independent, while the capacitor impedance, $Z_{C}$, changes with frequency according to

$$
Z_{C}=\frac{1}{i \omega C}
$$


where $i$ is the imaginary unit, $C$ the capacitance and $\omega$ the angular frequency. For a frequency, $f$, expressed in Hertz the angular frequency is found by

$$
\omega=2 \pi f
$$

Examples of typical circuit elements found in an experiment are electrolyte resistance, $R_{\mathrm{S}}$, and the electrical double layer at the electrode that can be represented by a capacitor, $C_{\mathrm{d} \text {. }}$. The equivalent circuit used to describe a lipid bilayer in this thesis is a capacitor, $C_{\mathrm{b}}$, and resistor, $R_{\mathrm{b}}$, in parallel (92). Equivalent circuits used to describe electrodes and electrodes with lipid bilayers are shown in Figure 5.3.

a

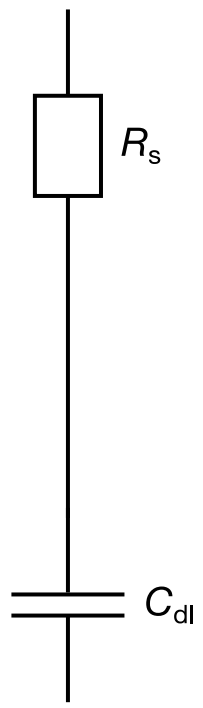

b

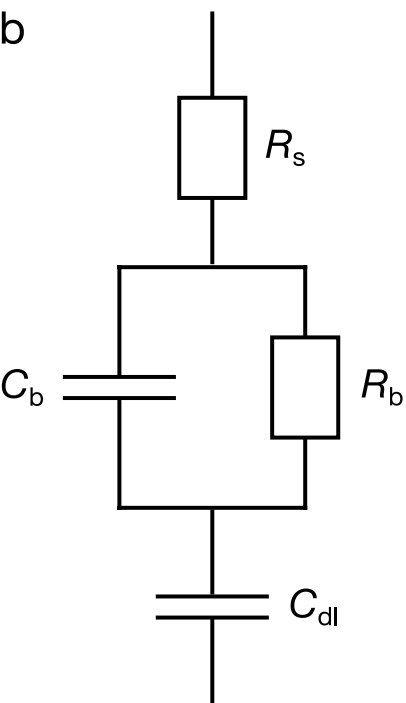

Figure 5.3 Equivalent circuits for a) electrode in electrolyte and b) electrode modified with a supported lipid bilayer.

The hydrophobic tails of the lipids block transport of ions dissolved in water and thus act as the dielectric in a capacitor sandwiched between the aqueous electrolyte on both sides. Imperfections in the bilayer can allow ions to cross the membrane. The parallel resistor represents the collection of these imperfections. Figure 5.2a shows Bode modulus curves of a gold electrode and a gold electrode with an added SLB. In order for the SLB to leave a mark on the impedance spectrum, its capacitance needs to be lower than the initial double layer capacitance (93). In the Bode modulus diagram, this lower capacitance will show as a higher impedance than the initial curve at high frequencies. The smaller the bilayer capacitance compared to the 
double layer capacitance, the further to the right in the graph the bilayer curve will deviate from the electrode curve. The bilayer resistance then determines at what frequency the curves join back together. Once the frequency has reached a low enough value to make the resistor the smallest impedance component in the bilayer circuit, the bilayer impedance becomes independent of frequency and the curve displays a horizontal section. At even lower frequencies, the impedance of the electrode double layer capacitor outpaces the bilayer impedance completely and the bilayer impedance curve merges with the electrode impedance curve. A higher bilayer resistance results in a larger vertical separation between the two curves, while a very small bilayer resistance can make the deviation difficult to distinguish. An SLB also leaves a signature in the phase shift. Generally, capacitively governed regions of the spectrum display a phase shift of $-90^{\circ}$, while resistively dominated regions do not display a phase shift $\left(0^{\circ}\right)$. In Figure $5.2 \mathrm{~b}$, the phase shift recorded for the bilayer differs from the bare electrode by not moving smoothly from $-90^{\circ}$ at the low end of the frequency spectrum to $0^{\circ}$ at the high end. Instead, the phase makes an additional turn back towards $-90^{\circ}$ around $10 \mathrm{kHz}$, indicating two capacitively dominated regions separated by one resistive.

\subsection{Atomic force microscopy}

The atomic force microscope (AFM) is a member of the scanning probe microscope (SPM) family. As the name suggests, an SPM uses a probe to scan a sample while recording interactions between the probe and the sample surface (94). The very first SPM was the scanning tunnelling microscope (STM), which measures the tunnelling current between probe and sample when the tip is biased. Next in line of SPM development was the AFM, which made it possible to study non-conducting samples and is operational in ambient conditions (95). The ability to measure in air and even in liquids has been of great importance for imaging biological samples (96). AFM gives information mainly about surface topography, but the setup can be modified to perform a vast line of measurements, such as mapping of magnetic properties (97) and work function (98).

At the heart of the AFM is a small flexible cantilever with a sharp tip facing the sample under investigation. Shining a laser on the cantilever, allowing the light to be reflected onto a photovoltaic detector, monitors bending of the cantilever. Before imaging, the laser detection system is aligned with equal intensity on both detector halves. Extension towards or retraction from the sample will offset the center of the beam and make the photo detector output a potential. Figure 5.4 shows an illustration of the detection system. 


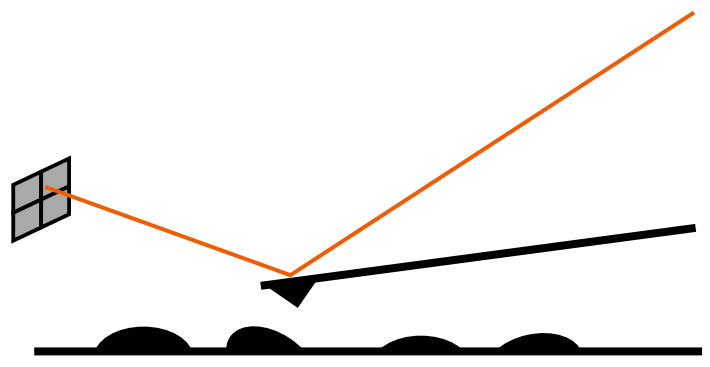

Figure 5.4 AFM detection system with laser shining on the cantilever. The deflection of the reflected beam is measured as it reaches the photovoltaic detector.

There are multiple ways to operate an AFM, each with its own advantages and limitations. The perhaps most intuitive operational mode is the contact mode. In contact mode the tip is in continuous contact with the sample surface, tracing groves and hills. The contact mode is also split into two categories - the fixed distance and the fixed deflection. To image a sample using fixed distance the scanner head holding the cantilever remains at a predetermined distance from the sample holder. The height profile is extracted from the deflection of the tip. In the case of fixed deflection, the scanner head is allowed to move in the zdirection to maintain a predefined deflection of the tip. The scanner position is used to reconstruct the sample profile. Contact mode gives good resolution but is the operational mode at the highest risk of damaging the sample under investigation.

To avoid the problems related to contact mode the AFM can also be operated in a non-contact mode. This keeps the tip on a safe distance from the sample and measures the interactions between tip and sample as the tip oscillates above the sample surface.

The third operational mode of AFM is the intermittent contact mode, also known as tapping mode. In this mode the tip cantilever oscillates in such a way that the tip contacts the surface in every oscillation. Compared to contact mode the intermittent contact mode is less destructive since the tip is in contact with the sample only for brief periods of time and does not drag along the sample surface. 


\subsection{Quartz crystal microbalance}

The quartz crystal microbalance (QCM) uses the oscillation of a piezoelectric crystal to measure mass change. Adding mass to the crystal lowers its resonance frequency, as illustrated in Figure 5.5a. The frequency response when adding rigid materials to the sensor follows the Sauerbrey relation:

$$
\frac{\Delta f}{f}=-\frac{\Delta m}{\rho A d}
$$

where $f$ is the eigenfrequency of the quartz crystal, and $\Delta f$ the change in eigenfrequency caused by the change in mass, $\Delta m$. $\rho, A$ and $d$ are the density, area and thickness of the quartz crystal plate, respectively (99). When studying biological systems, it is relevant to work with soft materials in aqueous environments. Soft materials in a viscous surrounding require more complicated equations and more data is needed to achieve good fits with the models (10o). For the work presented in this thesis a QCM setup with dissipation monitoring (QCM-D) was used. The dissipation is a measure of the dampening in the system (101). In the QCM-D the dissipation is monitored by the oscillation amplitude decay. A faster amplitude decay indicates a more dampened system. Figure $5.5 \mathrm{~b}$ shows examples of amplitude decays in two differently dampened systems.

a

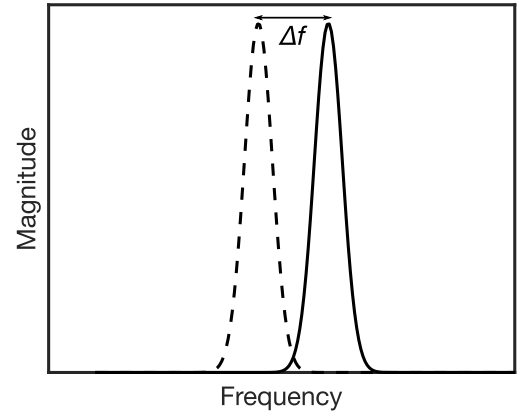

b
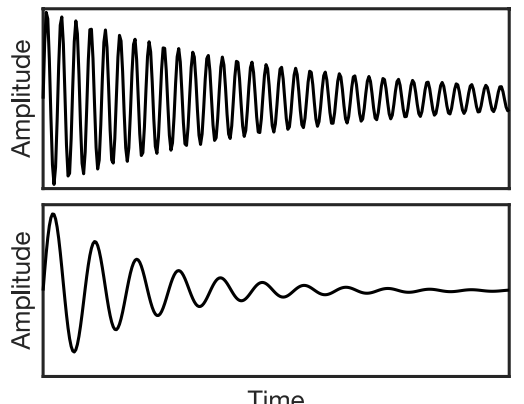

Figure $\mathbf{5 . 5}$ a) Resonance peak shift illustrating QCM measurement. b) Examples of amplitude decays recorded to determine dissipation. In the lower graph, the system has higher dissipation, as well as a lower resonance frequency than in the upper graph.

For work with lipid bilayers QCM-D can be used to show formation of the layer by the mass added on the crystal. A QCM-D tracking of POPC vesicle fusion, displayed in Figure 5.6, reveals the dynamics of bilayer formation with vesicles first adhering 
to the substrate causing the resonance frequency to decrease. At the same time the dissipation increases. When reaching a certain packing density, the vesicles rupture, releasing the water trapped inside them. This results in the frequency going back up, settling at a frequency shift of about $-23 \mathrm{~Hz}$ for the third overtone. As the water is released and the comparably large vesicles are replaced by a thin double layer the dissipation drops again and settles just above its initial value.

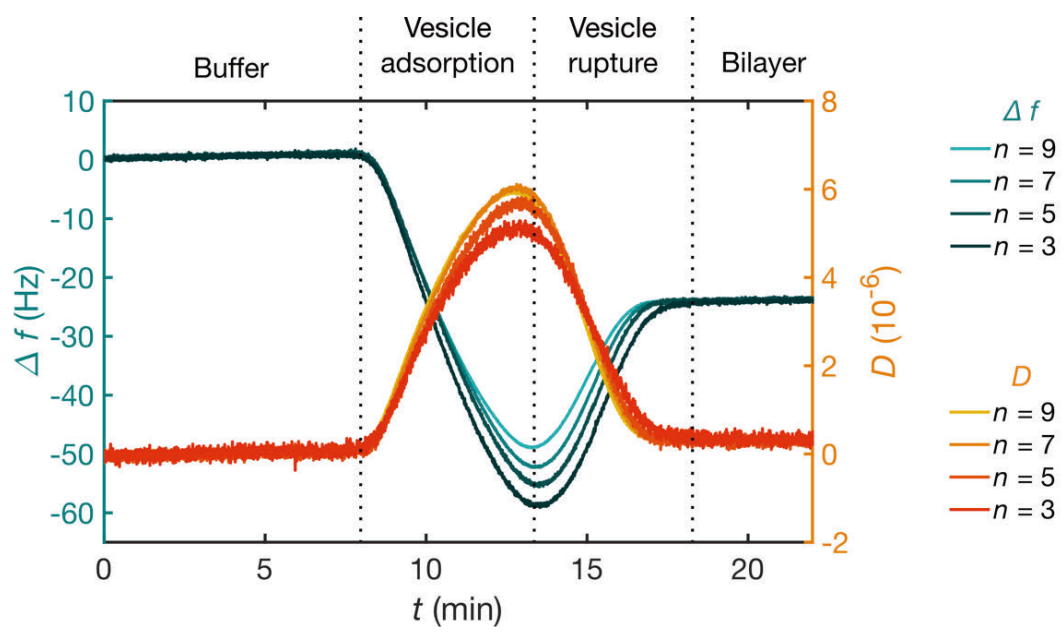

Figure 5.6 QCM-D recording of vesicle fusion experiment. The lipid used was POPC, at $0.2 \mathrm{mg} / \mathrm{ml}$ in PBS buffer. The experiment starts and ends with buffer flowing through the chamber (flow rate: $0.1 \mathrm{ml} / \mathrm{min}$ ).

The practical execution of a QCM experiment is usually done with the aid of a peristaltic pump that drives solution through a flow cell where the crystal is mounted. To simplify the interpretation of the data it is advantageous to use the same running buffer throughout the experiment. In the case of the vesicle fusion illustrated in Figure 5.6 the measurement starts and ends with buffer flowing through the cell. In the middle section where the vesicles are added, the same buffer flows through the cell, but it also contains vesicles suspended in the buffer. This way, the changes in frequency and dissipation can be assumed to originate mainly from events on the crystal surface and not the bulk solution.

QCM can be used in conjunction with other experiments. One example used in Paper III is QCM coupled with electrochemistry. The measurement is carried out as two experiments in parallel and is enabled by a special electrochemistry QCM cell. This cell allows for the sensor surface to be used as working electrode in an electrochemical setup. On the opposite side of the liquid within the cell there is a platinum electrode used as counter electrode. There is also the possibility to 
mount a reference electrode in the outlet channel. For investigating SLBs this instrument combination can be used to track how the impedance in the lipid bilayer evolves as it forms. Single frequency impedance tracking has shown that the bilayer impedance continues to increase after the vesicle fusion is complete (102).

\subsection{Vesicle extrusion}

For the publications included in this thesis, vesicles were prepared by extrusion. During the extrusion a lipid suspension is repeatedly pushed through a tracketched polycarbonate membrane with pore sizes in the nanometer range (103). The lipid suspension is prepared from a solution of lipids in an organic solvent, most commonly chloroform. As the chloroform is evaporated under a stream of nitrogen the lipids form sheets at the walls of the vessel. Aqueous buffer is then added to achieve the desired concentration of lipids. For the rehydration of the lipids some mechanical agitation is used, such as stirring or sonication. The resulting lipid suspension contains liposomes with a wide distribution of sizes. By extrusion of the liposomes, unilamellar vesicles with a narrow size distribution are formed (104). The lipid extruder is an assembly of two glass syringes where the suspension passes from the first syringe, through a filter membrane, into the second syringe. To ensure uniform vesicle sizes, the suspension is pushed back and forth multiple times between the syringes, finishing the procedure on the clean side of the filter membrane in the second syringe. Vesicles used in the work with this thesis were typically pushed a minimum of 11 times through a $200 \mathrm{~nm}$ pore size membrane, followed by at least 11 times through a $30 \mathrm{~nm}$ filter membrane. The resulting vesicle stock solution can be stored for a few days in the fridge, with dilution to the concentration desired for the experiment just prior to the start of the work.

\subsection{Fluorescence recovery after photobleaching}

One of the ways to demonstrate good diffusivity within the SLB is to use fluorescence recovery after photobleaching (FRAP). The technique takes advantage of something that is typically considered a weakness of fluorophores the photobleaching (105). Fluorescent molecules absorb light, and energy, in a process called excitation, and release the energy by emitting light of a longer wavelength than it absorbed. In a fluorescence microscopy setup, the excitation light is filtered out and only the emitted light reaches the eyepiece or detector. A fraction of the lipids used to form the bilayer are labelled with a fluorescent marker. Once the bilayer has formed, a region of the bilayer is photobleached by 
shining a high intensity light beam onto the lipids (106). The fluorescent markers in the illuminated region are then permanently bleached and the region appears dark in the image of the bilayer immediately after illumination. In a bilayer where the lipids are free to diffuse the bleached lipids will diffuse within and out of the region where they were located during the illumination. At the same time, lipids with fluorescent markers that were not bleached will diffuse into the bleached region, which results in healing of the bleached area. The sequence is illustrated in figure 5.7. From the dynamics of the healing the diffusivity of the lipids can be determined (107). Similar experiments can be conducted with fluorescent markers on proteins incorporated in the bilayer to study diffusion of proteins.
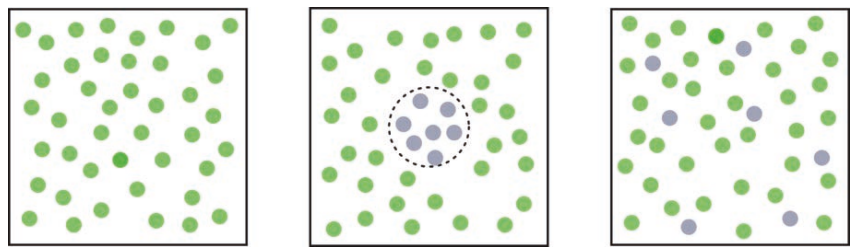

Figure 5.7 FRAP sequence where fluorophores (green) within the circle are bleached (grey) and then allowed to diffuse. Unbleached fluorophores diffuse into the bleached region.

\subsection{Electrical characterization of transistors}

To record a transistor's output characteristics and transfer curves two sourcemeasure-units (SMUs) are usually employed. An SMU can source a potential, while measuring the current, or vice versa. For the transistor measurements, one SMU sources the drain potential while measuring the drain current, and the other SMU sources the gate potential and measures the gate current. To obtain a transfer curve, the drain potential is set to a fixed value, while there is a sweep in the gate potential. For output characteristics, the drain potential is swept, while the gate is set to fixed values. Examples of OECT transfer curves and output characteristics can be found in Chapter 4 .

For frequency-dependence measurements, the transistor channels were connected in series with a resistor to create an amplifier circuit, as shown in Figure 5.8. The configuration was chosen to keep the potential applied between drain and gate at a minimum. The gate potential, $V_{\text {in }}$, was applied using a waveform generator, and a power supply delivered $V_{\mathrm{dd}}$. The output potential was then measured over the transistor using an oscilloscope. Simultaneously, the input potential was measured on another oscilloscope channel. Figure 5.9 shows 
examples of data recorded from the amplifier circuit at $10 \mathrm{~Hz}$ and $5 \mathrm{kHz}$. At $10 \mathrm{~Hz}$ the transistor provides the expected response; when the gate potential is high, the channel resistance increases and the magnitude of the drain potential, and $V_{\text {out }}$, increases. At $5 \mathrm{kHz}$, the transistor cannot keep up with the fast changes and the amplitude of $V_{\text {out }}$ has decreased and there is a phase shift between the two signals, meaning the peaks in $V_{\text {in }}$ and $V_{\text {out }}$ display a slight offset in time.

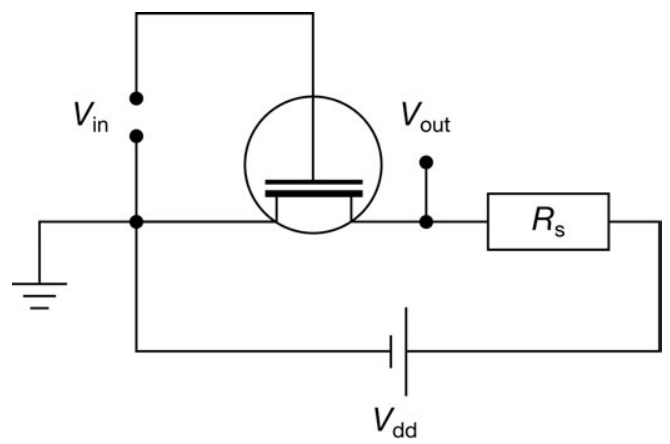

Figure 5.8 Amplifier circuit for dynamic transistor measurements.

a

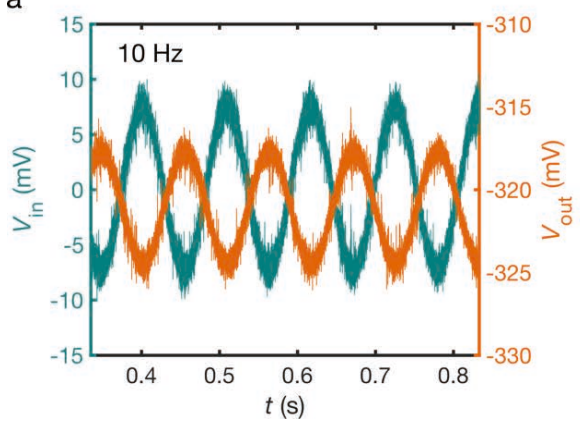

b

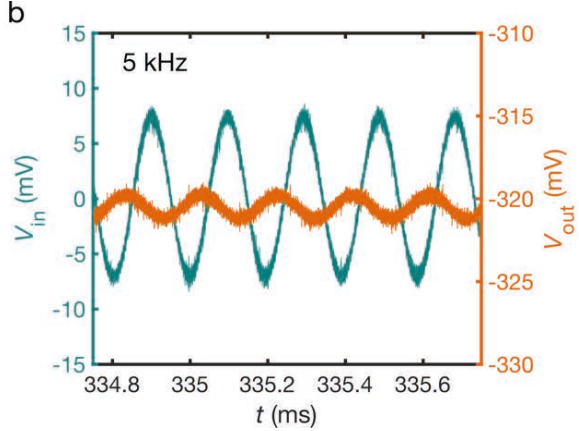

Figure 5.9 Amplifier circuit data with $V_{\text {in }}$ on left $y$-axes and $V_{\text {out }}$ on right $y$-axes. a) Input signal $10 \mathrm{~Hz}$. b) Input signal $5 \mathrm{kHz}$.

\subsection{Modelling}

Some of the results in this thesis, presented in Papers III and IV, were obtained from mathematical modelling. When modelling, a set of mathematical expressions are used to describe a phenomenon. The equations used here have been found by combination of other, previously known equations. When the equations governing the response of the system under investigation are found, the impact of changes to the equation variables can be studied. This way, the 
limitations of the system can be explored and favorable experimental conditions identified. In this work, the calculations have been performed in MATLAB. 


\section{Summary of papers}

This chapter will provide a brief summary of the most important results and conclusions from the papers included in the thesis.

\subsection{Paper I}

\section{Ferroelectric surfaces for cell release}

In this paper a ferroelectric film spin-coated on top of a thin indium tin oxide electrode was used as a cell release layer. The use of an electroactive cell release surface is an attractive alternative to enzymatic release methods that can otherwise impair cell adhesion in the next reseeding step. For this specific project, the target application was treatment of severe burn wounds, where transplantation of pre-confluent epithelial cells has been shown to be a promising technique. The goal was to show that cells can grow and develop healthily on the ferroelectric surface, and that cell release could be induced by polarization of the ferroelectric film. First, fibroblasts were seeded onto pristine ferroelectric films as well as films of both polarities. No morphological differences were observed between the cell cultures. The viability of cells grown on pristine P(VDF-TrFE) was then compared to that of cells grown on cell culture polystyrene, indicating a slight reduction in attachment and viability. To investigate the cell release properties of the film, fibroblasts were seeded onto unpolarized P(VDF-TrFE) and release was triggered by polarization events. A negative polarization induced release of a larger portion of the cells than positive polarization. After release the cells were reseeded to verify their viability. The cell release surface was found to function as intended, with cells being released and able to grow and proliferate after reseeding on cell culture polystyrene.

\subsection{Paper II}

Formation of supported lipid bilayers derived from vesicles of various compositional complexity on conducting polymer/silica surfaces

In Paper II, two material systems for promotion of vesicle fusion onto PEDOT:PSS were investigated. Vesicle fusion on PEDOT:PSS has been demonstrated previously, but the goal for this paper was to make the process faster, more reliable and compatible with a wider variety of lipids and lipid mixtures. 
The first material system was a composite material spin-coated on top of PEDOT:PSS from a blend of PEDOT:PSS and silica nanoparticles. The second material was a mesoporous silica film added on top of PEDOT:PSS. The mesoporous silica surface was also formed through spin-coating, from a mixture of tetraethylorthosilicate (TEOS), ethanol and hydrochloric acid. Pores were then created through extraction of the surfactant. Three methods of extraction were investigated, to verify complete extraction and ensure that the extraction process did not adversely affect the electrical properties of the PEDOT:PSS bottom layer. Extraction with the alcohols ethanol and methanol did not successfully remove the surfactant, which was apparent both from spectroscopy measurements and the electrical characterization. The method that did prove successful was extraction using UV/ozone exposure.

Both material systems were investigated with respect to electrical properties of the underlying polymer and the quality of the formed bilayers. The electrical characterization showed no major differences between the two top coatings, but the mesoporous silica showed more promising results for bilayer fluidity and portion of ruptured vesicles.

\subsection{Paper III}

The role of relative capacitances in impedance sensing with organic electrochemical transistors

In this paper the effects of the relationship between gate and channel capacitances on impedance OECT sensing were investigated. The results can be applied to sensing with supported lipid bilayers, either placed on the channel or on the gate, as well as to other types of capacitive modifications to the gate. The results in Paper III were obtained mainly by mathematical modelling. To describe the transistor's response to changes in capacitance the gating of the transistor was assumed to be linked to the potential drop over a channel capacitor. The charge on this capacitor determines the de-doping in the channel and thereby the channel current. If the potential applied between gate and channel is to drop elsewhere in the system, rather than over the channel capacitor, the channel will be less de-doped and the drain current larger than in the case where all of the applied potential drops over the channel capacitor.

The results are interpreted in the context of a depletion mode p-type OECT. First, the effect of a capacitance-limited gate was investigated, suggesting that gate capacitances more than 100 times larger than the channel capacitance would allow most of the applied potential to drop over the channel capacitor and contribute to the de-doping. Then, a scenario where sensing causes the gate 
capacitance to be reduced was studied. The sensor response was investigated with respect to $C_{\mathrm{g}} / C_{\mathrm{ch}}$ and the magnitude of the capacitance shift induced by the sensing event. Transistors that would typically be classified as good transistors, with large gates and thereby high transconductance, were found to not be very well suited for use as capacitive sensors. The results from the modelling show that small gate capacitances are required for sensing small capacitance shifts, while bigger gates can be used if the shift is expected to be large.

In the last modelled experiment, an equivalent circuit representing a supported lipid bilayer was added to the gate. The bilayer was represented by a capacitor and a resistor in parallel. Bilayer quality was assumed to mainly affect the resistance, and the bilayer capacitance was kept at a constant value. While the purely capacitive sensing occurs mainly at low frequencies, the bilayer sensing occurs in a frequency window set by the resistors and capacitors present in the circuit. In the lipid bilayer case, the sensor response was evaluated both with respect to the maximum response, and the total response in the investigated frequency range. Higher bilayer resistances result in sensing in a wider frequency window, and thereby also increases the total sensor response. Again, the high transconductance transistors were not the transistors exhibiting the largest sensor response. For small resistance bilayers, a small gate capacitance is needed to detect the lipids. For higher resistance layers, a higher gate capacitance can be used, increasing the maximum sensor response.

Finally, the findings were used to design a gold gate OECT able to detect the formation of a supported lipid bilayer.

\subsection{Paper IV}

\section{Expanding the understanding of organic electrochemical transistor function}

In Paper IV, the optimum biasing conditions for an OECT were investigated. The paper was mainly intended as a guide to improve signal strength for OECT-based sensors. The results in the paper were obtained through mathematical modelling, and then verified with experimental data. The model is an expansion of the Bernards model, where the potential range under investigation was extended into negative gate potentials (assuming a depletion mode p-type transistor). Positive drain potentials were left out of the analysis.

Five operational regimes were identified, out of which three are found for the negative gate potentials introduced in the paper. Each regime is governed by its own equation and is characterized by a unique combination of functional regions along the transistor channel: unaffected, de-doped and depleted. With the help of these equations, the drain current in a specific transistor was calculated for 
different drain and gate potentials. Transfer curves and corresponding transconductances were extracted from the data set and the transconductance curves were compared to experimental data.

The modelling showed that the transistor's pinch-off potential, $V_{\mathrm{p}}$, is the key to predicting transistor behavior and determine the best biasing conditions for the transistor. As the drain potential is lowered from o $\mathrm{V}$ to $-V_{\mathrm{p}}$, the transconductance increases. Once $-V_{\mathrm{p}}$ has been reached, the transconductance does not increase further. The model suggests that the transconductance is constant in the operational regime where it takes its highest value, but this is not the case for the actual transistor that displays a bell-shaped peak. The location of the experimental peak, however, aligns with the center of the modelled constant region. It was found that for any given drain potential, $V_{\mathrm{d}}$, the optimum gate potential, $V_{\mathrm{g}}$, will be $V_{\mathrm{g}}=1 / 2 V_{\mathrm{p}}+1 / 2 V_{\mathrm{d}}$. According to the model, the width of the region of constant transconductance narrows around $V_{\mathrm{d}}=-V_{\mathrm{p}}$, but is wider both at higher and lower drain potentials. The width of the constant regions seemed to also be reflected in the width of the experimental transconductance peak. If the transistor is used in an amplifier circuit, a constant transconductance means the amplifier can reproduce the input signal without distortion in a wider potential range. For capacitive sensing involving lipid bilayers, described in Paper III, the largest sensor signal will be obtained when operating the transistor at high transconductance 


\section{Concluding remarks}

\subsection{Bioelectronics}

During my time as a PhD student the field of bioelectronics has attracted more and more attention. Pharmaceutical companies have launched initiatives within electromedicine. The brain machine interface development has been given a proper push by Neuralink, a company founded by Elon Musk in 2016. The latest generation of the device, presented in August 2020, offers 1024 electrodes for read-and-write and has been granted status as breakthrough device by the American Food and Drug Administration (FDA). So far, the device has been implanted in pigs, and the plan is to start limited human trials in the near future.

The main challenges for devices attached on the skin or inside the body are probably going to remain the biocompatibility and signal strength over time for quite a while. Using soft and flexible materials have been advertised as a way to reduce the immunoresponse and limit scar tissue formation around electrodes, but this often means thin conductors and insulators, meaning less damage and corrosion needs to occur before functionality is lost.

\subsection{Ferroelectrics}

For the experiments presented in Paper I, the goal was to demonstrate a ferroelectric cell release surface. A gentle release method that does not cause harm to the cultured cells is still an attractive application to aim for. The advancements in tissue engineering will probably continue to increase the demand for gentle manipulation of cells and tissues when it is time to move between culture environments or into patients. From a practical standpoint, this particular release surface needs some further development to achieve a higher yield of devices. Pinholes were not discussed in the paper, but the efforts to limit their influence on the final device were a large part of the project and will be even more important for large area release surfaces.

The experiments in Paper I were targeting biological applications. However, the experimental device with a ferroelectric polymer sandwiched between a bottom electrode and an aqueous electrolyte could be used for studies of the ferroelectric material itself. The ease of removal and re-addition of what is effectively acting as the top electrode enables various types of imaging between polarization events. AFM imaging with piezo-force microscopy could show how the polarization is 
spatially distributed across the film surface (108). The technique could also be used to study ferroelectric depolarization (109).

\subsection{OECTs and lipid bilayers}

When I set out on this journey, I imagined the end result being a test bed for quick and accurate screening of potential drug candidates and their effects on their target membrane proteins. As time has passed, computing power has become cheaper and it is possible that the use of molecular dynamics simulations to simulate protein response in a virtual test bed is feasible before our physical device is ready for use. Indeed, towards the end of 2020 as this thesis is finalized, the results from the Critical Assessment of Structure Prediction (CASP) challenge came in. AlphaFold, an AI developed by DeepMind, was able to predict protein folding structure from the amino acid sequence with unprecedented accuracy (110). The advancements are predicted to have a major impact on protein research and drug discovery. Still, the technologies developed and presented in this thesis can find their use within other types of biosensors. With the trends moving towards more personalized medicine and more regular monitoring of a patient's health, the need for accurate and fast diagnostic tools will increase, and hopefully the lipid bilayer OECTs can be added to the toolbox. In the following paragraphs I will focus on the devices and suggest work that can be done in the near future to move the technology development forward.

One of the main goals with the research projects detailed in Papers II-IV was to integrate lipid bilayers into OECT-based biosensors. This goal has not yet been met in our lab, but now there is more knowledge and understanding guiding the decisions about device design, operation and lipid bilayer integration. Two ionpermeable material systems have been identified as possible additions to promote vesicle fusion on PEDOT:PSS. The electrical properties of the lipid bilayers formed on these surfaces need further characterization. It is likely, at least in the case of the silica particle blend, that the lipid bilayer capacitance turns out to be larger than the geometric area would indicate due to the increased surface area on the nano scale. Paper III suggests that for an OECT biosensor with a bilayer added to the channel it is important to ensure that the channel capacitance is sufficiently large to detect the added bilayer. A thicker channel will increase channel capacitance but keep the bilayer capacitance constant.

Another route of lipid incorporation touched upon in this thesis is addition of an SLB to the gate. With capacitive sensing using OECTs, as described in Paper III, it is possible to use a supported lipid bilayer on a metal gate. This approach would enable the use of thiol tethers to promote vesicle fusion on a gold gate, and also 
the use of spacers to allow adequate space for transmembrane proteins to function properly. Integration of a functional lipid bilayer with active proteins on a gold gate OECT is still to be demonstrated.

Incorporation of ion channels into supported lipid bilayers would mainly affect the bilayer's resistance. In Paper III the sensor response was assumed to be the response caused by the addition of the bilayer. In practice, when working with sensing based on channel proteins, the sensor response would be the alteration in the OECT's frequency response caused by the flow of ions through the channel protein, with the baseline being the frequency response with the bilayer but without ion openings. To construct a sensor with a large signal based on this system one should strive for a high resistance SLB, that turns into a low resistance SLB when the channel proteins allow ions through. This configuration would generate a response in a wide frequency window. Should the difference between the original resistance and the open channel resistance be small, it becomes even more important to pay attention to the frequency window of the sensing, since it is expected to become narrower.

In order to obtain a large sensor signal it is also important to operate the transistor in favorable conditions. In Paper III, the partial potential drop over the channel capacitor is used as an indicator of the overall transistor behavior. In practice, the sensory setup will probably utilize an amplifier circuit or a highfrequency SMU unit. In both cases an AC signal is applied at the gate, and the output is measured either as a variation in potential drop over a known resistor in series with the channel, or variation in channel current. Operation at favorable biasing conditions will make the fluctuations in channel resistance larger and harness the amplifying abilities of the OECT. Paper IV provides guidance on how to find the optimum operational point for each transistor, with the adapted Bernards model pointing out two operational regimes with high, constant transconductance. Operation at high transconductance is favorable not only for capacitive sensing, but for almost all OECT applications. The experimental data included in Paper IV shows that the transconductance is not constant but depends on the gate potential. The model indicates that the transconductance peak widens as the transistor is operated at negative gate potentials and drain potentials beyond the pinch-off potential.

Operation at constant transconductance would be ideal to achieve a wide linear regime for sensors and amplifiers, but second best is to operate where the transconductance peak is wide. For the transistor measured in Paper IV, the large potential differences between gate and drain required to achieve wide and high transconductance peaks for negative gate potentials lead to electrochemical reactions, which makes the model invalid and can cause damage to the transistor. 
A smaller pinch-off potential would enable operation in the negative gate regime while avoiding the electrochemical reactions associated with large potential differences. According to the Bernards model, the parameters determining the pinch-off potential are the initial charge carrier concentration, channel thickness and double layer capacitance. Tailoring of these material and device parameters to obtain OECTs with smaller pinch-off potentials opens for the possibility to operate the transistors at a high and wide transconductance peak. 


\section{References}

1. G. Kaushik, J. Leijten, A. Khademhosseini, Concise Review: Organ Engineering: Design, Technology, and Integration. Stem Cells. 35, 51-60 (2017).

2. C. E. Bouton, A. Shaikhouni, V. Nicholas, M. A. Bockbrader, D. A. Friedenberg, J. Mysiw, A. G. Morgan, D. M. Nielson, G. Sharma, P. B. Sederberg, B. C. Glenn, Restoring cortical control of functional movement in a human with quadriplegia. Nature. 533, 247-250 (2016).

3. D. C. Lu, V. R. Edgerton, M. Modaber, N. Auyong, E. Morikawa, S. Zdunowski, M. E. Sarino, M. Sarrafzadeh, M. R. Nuwer, R. R. Roy, Y. Gerasimenko, Engaging Cervical Spinal Cord Networks to Reenable Volitional Control of Hand Function in Tetraplegic Patients. Neurorehabil. Neural Repair. 30, 951-962 (2016).

4. J. Dunn, R. Runge, M. Snyder, Wearables and the medical revolution. Per. Med. 15, 429-448 (2018).

5. D. T. Simon, E. O. Gabrielsson, K. Tybrandt, M. Berggren, Organic Bioelectronics: Bridging the Signaling Gap between Biology and Technology. Chem. Rev. 116, 13009-13041 (2016).

6. A. Herland, K. M. Persson, V. Lundin, M. Fahlman, M. Berggren, E. W. H. Jager, A. I. Teixeira, Electrochemical Control of Growth Factor Presentation to Steer Neural Stem Cell Differentiation. Angew. Chemie. 50, 12529-33 (2011).

7. T. A. Sjöström, M. Berggren, E. O. Gabrielsson, P. Janson, D. J. Poxson, M. Seitanidou, D. T. Simon, A Decade of Iontronic Delivery Devices. Adv. Mater. Technol. 3, 1-10 (2018).

8. P. Lin, F. Yan, Organic Thin-Film Transistors for Chemical and Biological Sensing. Adv. Mater. 24, 34-51 (2012).

9. C. Liao, M. Zhang, M. Y. Yao, T. Hua, L. Li, F. Yan, Flexible Organic Electronics in Biology: Materials and Devices. Adv. Mater. 27, 7493-7527 (2015).

10. M. Berggren, D. Nilsson, N. D. Robinson, Organic materials for printed electronics. Nat. Mater. 6, 3-5 (2007).

11. J. Rivnay, S. Inal, A. Salleo, M. Berggren, G. G. Malliaras, Organic electrochemical transistors. Nat. Rev. Mater. 3, 1-14 (2018).

12. B. Alberts, D. Bray, K. Hopkin, A. Johnson, J. Lewis, M. Raff, K. Roberts, P. Walter, Essential Cell Biology (Garland Science, ed. 3, 2010).

13. T. Harayama, H. Riezman, Understanding the diversity of membrane lipid composition. Nat. Rev. Mol. Cell Biol. 19, 281-296 (2018).

14. M. Edidin, Shrinking patches and slippery rafts: scales of domains in the plasma. Trends Cell Biol. 11, 492-496 (2001).

15. F. H. Crick, On protein synthesis. Symp. Soc. Exp. Biol. 12, 138-163 (1958).

16. A. Krogh, B. Larsson, G. von Heijne, E. L. L. Sonnhammer, Predicting Transmembrane Protein Topology with a Hidden Markov Model: Application to Complete Genomes. J. Mol. Biol. 305, 567-580 (2001).

17. J. A. Killian, G. Von Heijne, How proteins adapt to a membrane - water 
interface. Trends Biochem. Sci. 25, 429-434 (2000).

18. D. Purves, G. J. Augustine, D. Fitzpatrick, W. C. Hall, A.-S. LaMantia, L. E. White, Neuroscience (Sinauer, ed. 5, 2012).

19. E. P. Widmaier, H. Raff, K. T. Strang, Vander's Human Physiology (McGraw-Hill, ed. 12, 2008).

20. P. Mueller, D. O. Rudin, H. T. Tien, W. C. Wescott, Methods for the Formation of Single Bimolecular Lipid Membranes in Aqueous Solution. J. Phys. Chem. 67, 534-535 (1963).

21. P. Mueller, D. O. Rudin, H. T. Tien, W. C. Wescott, Reconstitution of Cell Membrane Structure in vitro and its Transformation into an Excitable System. Nature. 194, 979-980 (1962).

22. P. L. Yeagle, The Membranes of Cells (Academic Press, ed. 3, 2016).

23. L. K. Tamm, H. M. McConnell, Supported phospholipid bilayers. Biophys. J. 47, 105-113 (1985).

24. T. Penkauskas, G. Preta, Biological applications of tethered bilayer lipid membranes. Biochimie. 157, 131-141 (2019).

25. D. Purves, G. J. Augustine, D. Fitzpatrick, W. C. Hall, A.-S. LaMantia, L. E. White, Neuroscience (Sinauer, ed. 5, 2012).

26. B. Alberts, D. Bray, K. Hopkin, A. Johnson, J. Lewis, M. Raff, K. Roberts, P. Walter, Essential Cell Biology (Garland Science, ed. 3, 2010).

27. S. EL Andaloussi, I. Mäger, X. O. Breakefield, M. J. A. Wood, Extracellular vesicles: biology and emerging therapeutic opportunities. Nat. Rev. Drug Discov. 12, 347-357 (2013).

28. R. Langer, New Methods of Drug Delivery. Science (80-. ). 249, 1527-1533 (1990).

29. A. A. Brian, H. M. McConnell, Allogeneic Stimulation of Cytotoxic T Cells by Supported Planar Membranes. Proc. Natl. Acad. Sci. U. S. A. 81, 6159-6163 (1984).

30. K. Kumar, C. S. Tang, F. F. Rossetti, M. Textor, B. Keller, J. Vo, E. Reimhult, Formation of supported lipid bilayers on indium tin oxide for dynamicallypatterned membrane-functionalized microelectrode arrays †. Lab Chip. 9, 718-725 (2009).

31. Y. Zhang, S. Inal, C. Hsia, M. Ferro, M. Ferro, S. Daniel, R. M. Owens, Supported Lipid Bilayer Assembly on PEDOT:PSS Films and Transistors. Adv. Funct. Mater. 26, 7304-7313 (2016).

32. Y. Shao, Y. Jin, J. Wang, L. Wang, F. Zhao, S. Dong, Conducting polymer polypyrrole supported bilayer lipid membranes. Biosens. Bioelectron. 20, 1373-9 (2005).

33. Y. Zhang, S. Wustoni, A. Savva, A. Giovannitti, I. Mcculloch, S. Inal, Lipid bilayer formation on organic electronic materials. J. Mater. Chem. C (2018), doi:10.1039/c8tc00370j.

34. D. Ohayon, C. Pitsalidis, A. Pappa, A. Hama, Y. Zhang, L. Gallais, R. M. Owens, Laser Patterning of Self-Assembled Monolayers on PEDOT:PSS Films for Controlled Cell Adhesion. Adv. Mater. Interfaces. 4, 1-8 (2017).

35. D. Axelrod, Cell-Substrate Contacts Illuminated by Total Internal Reflection Fluorescence. J. Cell Biol. 89, 141-145 (1981). 
36. S. R. Tabaei, J. Choi, G. H. Zan, V. P. Zhdanov, N. Cho, Solvent-Assisted Lipid Bilayer Formation on Silicon Dioxide and Gold. Langmuir, 10363-10373 (2014).

37. A. O. Hohner, M. P. C. David, J. O. Rädler, Controlled solvent-exchange deposition of phospholipid membranes onto solid surfaces. Biointerphases. 5, 1-8 (2010).

38. I. Langmuir, The constitution and fundamental properties of solids and liquids. II. Liquids. J. Am. Chem. Soc. 39, 1848-1906 (1917).

39. K. B. Blodgett, Films Built by Depositing Successive Monomolecular Layers. J. Am. Chem. Soc. 57, 1007-1022 (1935).

40. J. Liu, J. C. Conboy, Structure of a Gel Phase Lipid Bilayer Prepared by the Langmuir-Blodgett/Langmuir-Schaefer Method Characaterized by SumFrequency Vibrational Spectroscopy. Langmuir. 21, $9091-9097$ (2005).

41. J. R. Fried, in Polymer Science and Technology (Pearson Education, ed. 2, 2003), p. 1.

42. L. H. Baekeland, Method of Making Insoluble Products of Phenol and Formaldehyde (1909).

43. A century of ferroelectricity. Nat. Mater. 19, 129 (2020).

44. T. Soulestin, V. Ladmiral, F. Domingues, D. Santos, B. Améduri, Vinylidene fluoride- and trifluoroethylene-containing fluorinated electroactive copolymers . How does chemistry impact properties? Prog. Polym. Sci. 72, 1660 (2017).

45. T. Furukawa, Ferroelectric Properties of Vinylidene Fluoride Copolymers. Phase Transitions. 18, 143-211 (1989).

46. C. S. Hwang, T. Mikolajick, in Advances in Non-Volatile Memory and Storage Technology, B. Magyari-Köpe, Y. Nishi, Eds. (Elsevier Ltd., ed. 2, 2019), pp. 393-441.

47. A. Facchetti, $\pi$-Conjugated Polymers for Organic Electronics and Photovoltaic Cell Applications. Chem. Mater. 23, 733-758 (2011).

48. U. Ellervik, O. Sterner, Organisk kemi (Studentlitteratur, ed. 2, 2007).

49. G. Heywang, F. Jonas, Poly(alkylenedioxythiophenes) - New, Very Stable Conducting Polymers. Adv. Mater. 4, 116-118 (1992).

50. A. I. Hofmann, R. Kroon, S. Zokaei, E. Järsvall, C. Malacrida, S. Ludwigs, T. Biskup, C. Müller, Chemical Doping of Conjugated Polymers with the Strong Oxidant Magic Blue. Adv. Electron. Mater. 6, 1-8 (2020).

51. L. Groenendaal, F. Jonas, D. Freitag, H. Pielartzik, J. R. Reynolds, Its Derivatives: Past, Present, and Future. Adv. Mater. 12, 481-494 (2000).

52. D. Melling, J. G. Martinez, E. W. H. Jager, Conjugated Polymer Actuators and Devices: Progress and Opportunities. Adv. Mater. 31, 1-17 (2019).

53. J. Gladisch, E. Stavrinidou, S. Ghosh, A. Giovannitti, M. Moser, I. Zozoulenko, I. Mcculloch, M. Berggren, Reversible Electronic Solid-Gel Switching of a Conjugated Polymer. Adv. Sci. 7, 1-9 (2020).

54. Q. Pei, O. Inganäs, Conjugated Polymers and the Bending Cantilever Method: Elecrical Muscles and Smart Devices. Adv. Mater. 4, 3-4 (1992).

55. A. Maziz, A. Concas, A. Khaldi, J. Stålhand, N. Persson, E. W. H. Jager, Knitting and weaving artificial muscles. Sci. Adv. 3, 1-12 (2017). 
56. X. Crispin, F. L. E. Jakobsson, A. Crispin, P. C. M. Grim, P. Andersson, A. Volodin, C. Van Haesendonck, M. Van Der Auweraer, W. R. Salaneck, M. Berggren, The Origin of the High Conductivity of ( PEDOT - PSS ) Plastic Electrodes. Chem. Mater. 18, 4354-4360 (2006).

57. H. Shi, C. Liu, Q. Jiang, J. Xu, Effective Approaches to Improve the Electrical Conductivity of PEDOT:PSS: A Review. Adv. Electron. Mater. 1, 1-16 (2015).

58. J. Y. Kim, J. H. Jung, D. E. Lee, J. Joo, Enhancement of electrical conductivity of poly(3,4-ethylenedioxythiophene)/poly(4-styrenesulfonate) by a change of solvents. Synth. Met. 126, 311-316 (2002).

59. J. Nevrela, M. Micjan, M. Novota, S. Kovacova, M. Pavuk, P. Juhasz, J. K. Jr, J. Jakabovic, M. Weis, Secondary Doping in Poly(3, 4-ethylenedioxythiophene): Poly(4-styrenesulfonate) Thin Films. Polym. Phys. 53, 1139-1146 (2015).

60. H. L. von Helmholtz, Some Laws Concerning the Distribution of Electric Currents in Volume Conductors With Applications to Experiments on Animal Electricity (reprint). Proc. IEEE. 92, 868-870 (2004).

61. A. V Volkov, K. Wijeratne, E. Mitraka, U. Ail, D. Zhao, K. Tybrandt, J. W. Andreasen, M. Berggren, X. Crispin, I. V Zozoulenko, Understanding the Capacitance of PEDOT:PSS. Adv. Funct. Mater. 27, 1-10 (2017).

62. I. Sahalianov, S. Kumar Singh, K. Tybrandt, M. Berggren, I. Zozoulenko, The intrinsic volumetric capacitance of conducting polymers: pseudo-capacitors or double-layer supercapacitors? RSC Adv. 9, 42498-42508 (2019).

63. J. Rivnay, P. Leleux, M. Ferro, M. Sessolo, A. Williamson, D. A. Koutsouras, D. Khodagholy, M. Ramuz, X. Strakosas, R. M. Owens, C. Benar, J. Badier, C. Bernard, G. G. Malliaras, High-performance transistors for bioelectronics through tuning of channel thickness. Sci. Adv. 1, 1-6 (2015).

64. M. Guarnieri, Seventy Years of Getting Transistorized. IEEE Ind. Electron. Mag. 11, 33-37 (2017).

65. J. Bardeen, W. Brattain, The Transistor, a Semi-Conductor Triode. Phys. Rev. 74, 231-232 (1948).

66. J. A. Morton, W. J. Pietenpol, The Technological Impact of Transistors. Proc. IRE. 46, 955-959 (1958).

67. H. S. White, G. P. Kittlesen, M. S. Wrighton, Chemical Derivatization of an Array of Three Gold Microelectrodes with Polypyrrole : Fabrication of a MoleculeBased Transistor. J. Am. Chem. Soc. 106, 5375-5377 (1984).

68. G. Tarabella, M. Villani, D. Calestani, R. Mosca, S. Iannotta, A. Zappettini, N. Coppedé, A single cotton fiber organic electrochemical transistor for liquid electrolyte saline sensing. J. Mater. Chem. 22, 23830-23834 (2012).

69. S. Zhang, Y. Chen, H. Liu, Z. Wang, H. Ling, C. Wang, J. Ni, B. Çelebi-saltik, X. Wang, X. Meng, H. Kim, A. Baidya, S. Ahadian, N. Ashammakhi, M. R. Dokmeci, J. Travas-sejdic, A. Khademhosseini, Room-temperature-formed PEDOT:PSS hydrogels rnable injectable, soft, and healable organic bioelectronics. Adv. Mater. 32, 1-7 (2020).

70. E. Stavrinidou, R. Gabrielsson, E. Gomez, X. Crispin, O. Nilsson, D. T. Simon, M. Berggren, Electronic plants. Sci. Adv. 1, 1-8 (2015).

71. B. D. Nilsson, M. Chen, T. Kugler, T. Remonen, M. Armgarth, M. Berggren, Bistable and Dynamic Current Modulation in Electrochemical Organic Transistors. Adv. Mater. 14, 51-54 (2002). 
72. R. Colucci, H. Frulani, D. P. Barbosa, F. Günther, P. Cavassin, G. C. Faria, Recent advances in modeling organic electrochemical transistors. Flex. Print. Electron. 5 (2020), doi:10.1088/2058-8585/ab601b.

73. D. A. Bernards, G. G. Malliaras, Steady-State and Transient Behavior of Organic Electrochemical Transistors. Adv. Funct. Mater. 17, 3538-3544 (2007).

74. G. C. Faria, D. T. Duong, A. Salleo, On the transient response of organic electrochemical transistors. Org. Electron. 45, 215-221 (2017).

75. G. C. Faria, D. T. Duong, A. Salleo, C. a. Polyzoidis, S. Logothetidis, J. Rivnay, R. Owens, G. G. Malliaras, Organic electrochemical transistors as impedance biosensors. MRS Commun. 4, 189-194 (2014).

76. J. T. Friedlein, J. Rivnay, D. H. Dunlap, I. Mcculloch, S. E. Shaheen, R. R. McLeod, G. G. Malliaras, Influence of disorder on transfer characteristics of organic electrochemical transistors. Appl. Phys. Lett. 111 (2017), doi:10.1063/1.4993776.

77. D. Tu, S. Fabiano, Mixed ion-electron transport in organic electrochemical transistors. Appl. Phys. Lett. 117 (2020), doi:10.1063/5.0012599.

78. K. Tybrandt, I. V Zozoulenko, M. Berggren, Chemical potential-electric double layer coupling in conjugated polymer-polyelectrolyte blends. Sci. Adv. 3, 1-7 (2017).

79. Z. M. Szymanski, D. Tu, R. Forchheimer, 2-D Drift-Diffusion Simulation of Organic Electrochemical Transistors. IEEE Trans. Electron Devices. 64, 51145120 (2017).

80. X. Strakosas, M. Bongo, The organic electrochemical transistor for biological applications. J. Appl. Polym. Sci. 132 (2015), doi:10.1002/app.41735.

81. M. Berto, C. Diacci, L. Theuer, M. Di Lauro, D. T. Simon, Label free urea biosensor based on organic electrochemical transistors. Flex. Print. Electron. 2 (2018).

82. C. Diacci, J. W. Lee, P. Janson, G. Dufil, G. Méhes, M. Berggren, D. T. Simon, E. Stavrinidou, Real-Time Monitoring of Glucose Export from Isolated Chloroplasts Using an Organic Electrochemical Transistor. Adv. Mater. Technol. 5 (2020), doi:10.1002/admt.201900262.

83. L. H. Jimison, S. A. Tria, D. Khodagholy, M. Gurfi, E. Lanzarini, A. Hama, G. G. Malliaras, R. M. Owens, Measurement of Barrier Tissue Integrity with an Organic Electrochemical Transistor. Adv. Mater. 24, 5919-5923 (2012).

84. D. A. Bernards, G. G. Malliaras, G. E. S. Toombes, S. M. Gruner, Gating of an organic transistor through a bilayer lipid membrane with ion channels. Appl. Phys. Lett. 89, 10-13 (2006).

85. D. Khodagholy, T. Doublet, P. Quilichini, M. Gurfinkel, P. Leleux, A. Ghestem, E. Ismailova, T. Herve, C. Bernard, G. G. Malliaras, In vivo recordings of brain activity using organic transistors. Nat. Commun. 4 (2013), doi:10.1038/ncomms2573.

86. O. Yaghmazadeh, F. Cicoira, D. A. Bernards, S. Y. Yang, Y. Bonnassieux, G. G. Malliaras, Optimization of Organic Electrochemical Transistors for Sensor Applications. J. Polym. Sci. Part B Polym. Phys., 34-39 (2010).

87. D. L. Smith, Thin-Film Depostion - Principles and Practice (McGraw-Hill, 1995).

88. K. Norrman, N. B. Larsen, 6 Studies of spin-coated polymer films. Annu. Reports Prog. Chem. Sect. C Phys. Chem. 101, 174-201 (2005). 
89. G. S. May, S. M. Sze, Fundamentals of Semiconductor Fabrication (Wiley, 2004).

90. G. S. May, S. M. Sze, Fundamentals of Semiconductor Fabrication (Wiley, 2004).

91. E. P. Randviir, C. E. Banks, Electrochemical impedance spectroscopy: an overview of bioanalytical applications. Anal. Methods, 1098-1115 (2013).

92. C. Steinem, a Janshoff, W. P. Ulrich, M. Sieber, H. J. Galla, Impedance analysis of supported lipid bilayer membranes: a scrutiny of different preparation techniques. Biochim. Biophys. Acta. 1279, 169-80 (1996).

93. J. Lin, M. Merzlyakov, K. Hristova, P. C. Searson, Impedance spectroscopy of bilayer membranes on single crystal silicon. Biointerphases. 3, FA33 (2008).

94. S. M. Salapaka, M. V. Salapaka, Scanning probe microscopy. IEEE Conrol Syst. Mag. 28, 65-83 (2008).

95. C. Binnig, C. F. Quate, C. Gerber, Atomic force microscope. Phys. Rev. Lett. 56, 930-933 (1986).

96. S. Kasas, N. H. Thomson, B. L. Smith, P. K. Hansma, J. Miklossy, H. G. Hansma, Biological Applications of the AFM: From Single Molecules to Organs. Int. J. Imaging Syst. Technol. 8, 151-161 (1997).

97. Y. Martin, H. K. Wickramasinghe, Magnetic imaging by '“force microscopy"' with 1000 Å resolution. Appl. Phys. Lett. 50, 1455-1457 (1987).

98. C. Sommerhalter, T. W. Matthes, T. Glatzel, A. Jäger-Waldau, M. C. Lux-Steiner, High-sensitivity quantitative Kelvin probe microscopy by noncontact ultrahigh- vacuum atomic force microscopy. Appl. Phys. Lett. 75, 10-13 (1999).

99. G. Sauerbrey, Verwendung von Schwingquarzen zur Wägung dünner Schichten und zur Mikrowägung. Zeitschrift für Phys. 115, 206-222 (1959).

100. M. C. Dixon, Quartz Crystal Microbalance with Dissipation Monitoring: Enabling Real-Time Characterization of Biological Materials and Their Interactions. J. Biomol. Tech. 19, 151-158 (2008).

101. E. Reimhult, C. Larsson, B. Kasemo, F. Ho, Simultaneous Surface Plasmon Resonance and Quartz Crystal Microbalance with Dissipation Monitoring Measurements of Biomolecular Adsorption Events Involving Structural Transformations and Variations in Coupled Water. Anal. Chem. 76, 72117220 (2004).

102. E. Briand, M. Zäch, S. Svedhem, B. Kasemo, S. Petronis, Combined QCM-D and EIS study of supported lipid bilayer formation and interaction with poreforming peptides. Analyst. 135, 343-50 (2010).

103. M. J. Hope, M. B. Bally, G. Webb, P. R. Cullis, Production of large unilamellar vesicles by a rapid extrusion procedure. Characterization of size distribution, trapped volume and ability to maintain a membrane potential. Biochim. Biophys. acta - Biomembr. 812, 55-56 (1985).

104. B. J. Frisken, C. Asman, P. J. Patty, Studies of Vesicle Extrusion. Langmuir. 16, 928-933 (2000).

105. K. Jacobson, E. Wu, G. Poste, Measurement of the translation mobility of concanavalin a in glycerol-saline solutions and on the cell surface by fluorescence recovery after photobleaching. Biochim. Biophys. acta Biomembr. 433, 215-222 (1976).

106. E.-S. Wu, K. Jacobson, D. Papahadjopoulos, Lateral Diffusion in Phospholipid Multibilayers Measured by Fluorescence Recovery after Photobleaching. Biochemistry. 16, 3936-3941 (1977). 
107. P. Jönsson, M. P. Jonsson, J. O. Tegenfeldt, F. Höök, A Method Improving the Accuracy of Fluorescence Recovery after Photobleaching Analysis. Biophys. J. 95, 5334-5348 (2008).

108. S. V Kalinin, D. A. Bonnell, Imaging mechanism of piezoresponse force microscopy of ferroelectric surfaces. Phys. Rev. B. 65, 1-11 (2002).

109. D. Zhao, T. Lenz, G. H. Gelinck, P. Groen, D. Damjanovic, D. M. De Leeuw, I. Katsouras, Depolarization of multidomain ferroelectric materials. Nat. Commun. 10, 1-11 (2019).

110. E. Callaway, "It will change everything": AI makes gigantic leap in solving protein structures. Nature. 588, 203-204 (2020). 

9 Papers 


\section{Papers}

The papers associated with this thesis have been removed for copyright reasons. For more details about these see:

http://urn.kb.se/resolve?urn=urn:nbn:se:liu:diva-171993 


\section{FACULTY OF SCIENCE AND ENGINEERING}

Linköping Studies in Science and Technology, Dissertation No. 2118, 2021 Department of Science and Technology

Linköping University

SE-581 83 Linköping, Sweden

www.liu.se 